\title{
Balance equations in semi-relativistic quantum hydrodynamics
}

\author{
A Yu Ivanov, P A Andreev, L S Kuz'menkov \\ Faculty of Physics, Moscow State University, Leninskie Gory build. 1 struct. 2, \\ Moscow, 119991, Russian Federation \\ E-mail: alexmax1989@mail.ru
}

\begin{abstract}
Аннотация. Method of the quantum hydrodynamics has been applied in quantum plasmas studies. As the first step in our consideration, derivation of classical semi-relativistic (i. e. described by the Darwin Lagrangian on microscopic level) hydrodynamical equations is given after a brief review of method development. It provides better distinguishing between classic and quantum semi-relativistic effects. Derivation of the classical equations is interesting since it is made by a natural, but not very widespread method. This derivation contains explicit averaging of the microscopic dynamics. Derivation of corresponding quantum hydrodynamic equations is presented further. Equations are obtained in the five-momentum approximation including the continuity equation, Euler and energy balance equations. It is shown that relativistic corrections lead to presence of new quantum terms in expressions for a force field, a work field etc. The semi-relativistic generalization of the quantum Bohm potential is obtained. Quantum part of the energy current, which is an analog of the quantum Bohm potential for the energy evolution equation, is derived. The Langmuir wave dispersion in semi-relativistic quantum plasmas, corresponding to the Darwin Lagrangian, is also considered to demonstrate contribution of semi-relativistic effects on basic plasma phenomenon.
\end{abstract}

\section{Introduction}

Paper is dedicated to further development of the many-particle quantum hydrodynamics [1-[4]. In previous works on this subject non-relativistic systems of particles and also influence of several (particularly, spin related) relativistic corrections in Hamiltonian describing system have been studied in [2], 4], [5]. Main goal of this work is an obtaining of the quantum hydrodynamical equations for system of charged particles with the Coulomb and the current-current interactions, placed into an external electromagnetic field. The relativistic correction to the kinetic energy of particles is also taken into consideration. Historically the Biot-Savart-Laplace law was among the first examples of the current-current interaction. It is possible to formulate the problem with electromagnetic interaction of $\mathrm{N}$ particles. Lagrangian of this system in semi-relativistic 
approximation to the second order of $v / c$ was derived by Darwin [6] and has form

$$
\begin{aligned}
& L=\sum_{i=1}^{N}\left(\frac{m_{i} v_{i}^{2}}{2}+\frac{m_{i} v_{i}^{4}}{8 c^{2}}\right)-\frac{1}{2} \sum_{i, j=1, i \neq j}^{N} \frac{e_{i} e_{j}}{r_{i j}} \\
& +\sum_{i, j=1, i \neq j}^{N} \frac{e_{i} e_{j}}{4 c^{2}}\left[\frac{\mathbf{v}_{i} \mathbf{v}_{j}}{r_{i j}}+\frac{\left(\mathbf{v}_{i} \mathbf{r}_{i j}\right)\left(\mathbf{v}_{j} \mathbf{r}_{i j}\right)}{r_{i j}^{3}}\right] .
\end{aligned}
$$

where $m_{i}, e_{i}$ are masses and charges of particles, $c$ is the speed of light, $\mathbf{r}_{i}$ is radius vector of $i$-th particle, $\mathbf{v}_{i}=\dot{\mathbf{r}}_{i}$ are velocities of particles, $\mathbf{r}_{i j}=\mathbf{r}_{i}-\mathbf{r}_{j}$. The first group of terms in the first line is the non-relativistic kinetic energy of particles, the second group is associated with the relativistic correction to it, the third is energy of the Coulomb interaction. Group of terms in the second line correspond to the currentcurrent interaction. Radiation of electromagnetic waves is not included into Lagrangian in this approximation.

Kinetic equation describing dynamics of $\mathrm{N}$ charged interacting particles in selfconsistent field approximation was proposed by A. A. Vlasov [7], 8] in 1938. In the process full electromagnetic interaction was taken into account, i. e. fields produced by particles satisfy to the full set of Maxwell equations. Derivation of a kinetic equation for $\mathrm{N}$ particle system with the Coulomb interaction was presented in 1946 by N. N. Bogolyubov, it is known as the BBGKY hierarchy (the method can be found in [9], [10]). Derivation of the kinetic equation based on the Darwin Lagrangian presented in [11], [12], [13], some further development of this field can be found in Refs. [14]-[17]. Consideration of the semi-relativistic effects in quantum plasmas was started in Refs. [18]-[22], some of it was done in terms of the Wigner quantum distribution function [23]. Recent papers show that there is strong interest to relativistic effects in plasmas [24]-[31] and quantum plasmas (see for instance Ref. [32]). The semi-relativistic corrections be of interest also in equations of quantum plasma kinetics [32]. The spin-orbit interaction in quantum plasmas was considered in [4], 333]- 36]. Quantum hydrodynamics based on the relativistic Klein-Gordon equations was considered in 37. Quantum hydrodynamical description of the Dirac electron was presented in [38]. Many astrophysical applications wait for creation of relativistic quantum hydrodynamics and kinetics. Some of these astrophysical phenomena were described in recent review [39].

Some properties of the semi-relativistic many-particle QHD have been considered in our recent papers [40] and [41. These research were focused on the Euler equation and Langmuir wave dispersion. The Darwin interaction was also considered in [40]. It was shown that the Darwin interaction competes with contribution given by the semirelativistic part of the kinetic energy.

Creation of fully relativistic quantum hydrodynamics meets some complications, because quantum equation describing dynamics of $\mathrm{N}$ relativistic particles with full electromagnetic interaction does not exist. This system of particles is non-Hamiltonian system. Evolution of one particle with spin in external electromagnetic field is described by the Dirac equation.

Quantum generalization of the Hamiltonian corresponding to the Darwin 
Lagrangian was proposed by Breit [42]. So, on the way of relativistic quantum hydrodynamics construction is naturally to use this Hamiltonian describing quantum system of $\mathrm{N}$ particles with electromagnetic interaction to the second order of $v / c$. Quantum mechanical derivation of the Breit Hamiltonian was done by L. D. Landau; derivation from scattering amplitude of two electrons is presented in Ref. [43] (see section 83). The Breit Hamiltonian has form

$$
\begin{aligned}
& \hat{H}=\sum_{i=1}^{N}\left(\frac{\hat{\mathbf{p}}_{i}^{2}}{2 m_{i}}-\frac{\hat{\mathbf{p}}_{i}^{4}}{8 m_{i}^{3} c^{2}}\right) \\
& +\frac{1}{2} \sum_{i, j=1, i \neq j}^{N}\left\{\frac{e_{i} e_{j}}{r_{i j}}-\pi \frac{e_{i} e_{j} \hbar^{2}}{2 c^{2}}\left(\frac{1}{m_{i}^{2}}+\frac{1}{m_{j}^{2}}\right) \delta\left(\mathbf{r}_{i}-\mathbf{r}_{j}\right)\right. \\
& -\frac{e_{i} e_{j}}{2 m_{i} m_{j} c^{2} r_{i j}}\left(\hat{\mathbf{p}}_{i} \hat{\mathbf{p}}_{j}+\frac{\mathbf{r}_{i j}\left(\mathbf{r}_{i j} \hat{\mathbf{p}}_{i}\right) \hat{\mathbf{p}}_{j}}{r_{i j}^{2}}\right) \\
& -\frac{e_{i} e_{j} \hbar}{4 c^{2} r_{i j}^{3}}\left[\frac{\left[\mathbf{r}_{i j} \hat{\mathbf{p}}_{i}\right]_{\alpha} \sigma_{i \alpha}}{m_{i}^{2}}-\frac{\left[\mathbf{r}_{i j} \hat{\mathbf{p}}_{j}\right]_{\alpha} \sigma_{j \alpha}}{m_{j}^{2}}+\frac{2\left(\left[\mathbf{r}_{i j} \hat{\mathbf{p}}_{i}\right]_{\alpha} \sigma_{j \alpha}-\left[\mathbf{r}_{i j} \hat{\mathbf{p}}_{j}\right]_{\alpha} \sigma_{i \alpha}\right)}{m_{i} m_{j}}\right] \\
& \left.+\frac{e_{i} e_{j} \hbar^{2}}{8 m_{i} m_{j} c^{2}} \sigma_{i \alpha} \sigma_{j \beta}\left[\frac{\delta^{\alpha \beta}}{r_{i j}^{3}}-\frac{3 x_{\alpha i j} x_{\beta i j}}{r_{i j}^{5}}-\frac{8 \pi}{3} \delta^{\alpha \beta} \delta\left(\mathbf{r}_{i}-\mathbf{r}_{j}\right)\right]\right\},
\end{aligned}
$$

where $\hat{\mathbf{p}}_{i}=-i \hbar \nabla_{i}$ is momentum operator of particle, $\sigma_{i \alpha}$ is spin operator of particle, $\hbar$ is the Planck constant. As it is seen from this expression, the Breit Hamiltonian contains, along with the Coulomb and the current-current interactions, also the spinspin (the fourth line), spin-current and spin-orbit interactions (the third line). There is also interaction proportional to $\delta\left(\mathbf{r}_{i}-\mathbf{r}_{j}\right)$, which corresponds to the Zitterbewegung effect.

Through studies of specific physical processes, such as dispersion of waves, interaction of charged beams with plasmas, equations of quantum hydrodynamics have been used in modern literature, and these equations can be derived from both the manyparticle Schrödinger equation to be truncated for particular system of particles [1], [2], [3], 44] and from the Schrödinger equation for one particle in an external field [45]-47].

Since we develop the method of quantum hydrodynamics, let us briefly mention history of its development. In 1926 Madelung [48], on account of the Schrödinger equation for one particle in an external field, derived a set of equation in hydrodynamical form. This set of equations consists from the continuity equation and the momentum balance equation. Velocity field is potential there. In this way, Madelung made a transfer from one abstract complex function to the observable physical quantities - probability density and velocity field. Hydrodynamical formulation of quantum problem on motion of charged particle with a spin in an external electromagnetic field was accomplished by Takabayashi in 1955 [49]. In his work, along with the continuity and momentum balance equations, an equation of spin evolution was obtained. An explicit form of the force field associated with magnetic moment interaction with an external field was derived in the momentum balance equation. So hydrodynamics may appear directly from equations of mechanics. Nevertheless, the traditional way of derivation of hydrodynamic equation is getting of moments of kinetic equation. To the end one can use the Wigner kinetic 
equation [10], [19], 23], [50]. However, it is possible to find straightforward derivation of quantum hydrodynamics from many-particle Schrödinger equation. It was done in 19992001 in Refs. [1], [2], [51]. Further development was made in Refs. [3], [5], 41]. Quantum hydrodynamics has different applications, but most of all it has been used for studying of quantum plasmas. Reviews of recent applications of the quantum hydrodynamics for quantum plasmas were presented in Refs. [52], [53].

In Ref. 54 effectiveness of hydrodynamical description (obtaining from the one-particle Schrödinger equation) for kinetic properties of quantum plasmas was demonstrated.

Investigating quantum plasma of many-electron atom, many-nucleon problem, transport phenomena, propagation modes we have to deal with dynamics of many interacting particles. Schrödinger equation for a system of $\mathrm{N}$ interacting particles is defined in a $3 \mathrm{~N}$ dimensional configuration space, when propagation of excitations, processes of momentum and energy exchange are taken place in three dimensional physical space. So, there is problem of conversion from function defined in physical space to an equivalent quantum description of system in terms of physical fields in three dimensional space. The method of many-particle quantum hydrodynamics [1], [2], [3] solves this problem allowing to get dynamics in physical space. This transition is reached by the Dirac delta function existing in definition of hydrodynamic variables. They play role of projection operators from $3 \mathrm{~N}$ dimensional configurational space to three dimensional physical space.

In the first paper on [1] quantum hydrodynamic method for $\mathrm{N}$ interacting particles the Coulomb interaction only is taken into account. Many-particle QHD allows to derive the continuity and Euler equations, but it also allows to derive other hydrodynamic equations, such as the energy, pressure, energy flux evolution equations, and make truncation at necessary point. The energy evolution equation were derived in Ref. [1]. Quantum contributions in the momentum and energy balance equations were obtained. Quantum exchange correlations for bosons and fermions were considered in [1] as well. It was done to consider interparticle interaction beyond the self-consistent field approximation.

In following papers other interactions were included in the QHD scheme: the spinspin interaction [2], the spin-current interaction [4], [5], and the spin-orbit interaction [4], [33], [34]. Exchange part of the Coulomb and spin-spin interactions were derived in Ref. [51. It was applied for calculation of wave dispersion in quantum plasmas [55]. In other papers, the ultra-cold quantum gases of neutral particles [44], systems of particles with the electric dipole-dipole interactions, where equations of polarization evolution were derived and applied [3], [56], system of spinning particles with electric dipole interactions [36] etc were investigated. We should mention that QHD theory of the ultra-cold quantum gases of neutral particles is based upon the exchange part of the short-range interaction [44].

Evolution of magnetic moments in quantum plasmas leads to different phenomena [52. Particularly it supports propagation of new type of excitations [57], [58], [59], where 
spin dynamics are involved in evolution of electromagnetic perturbations. So we can call them spin-electromagnetic plasma waves. Existence of specific spin waves, where the electric field existing in plasma gives no contribution in mechanism of propagation of these spin waves, was also shown in Ref. [57]. More detailed description of the spin waves and spin-electromagnetic plasma waves can be found in Ref. 44. Instabilities of magnetized quantum plasmas can be caused by propagation of neutron beam [4], 55]. These instabilities are caused by the spin-spin interaction of neutron spins and plasma spins, and also the spin-current interaction of neutron beam spins and electric currents in plasma.

Some papers dedicated to discussion of applicability area for the QHD have been appeared [60], [61]. We should mention that these debates are conducted around the QHD equations obtained from the one-particle Schrödinger equation or the manyparticle Schrödinger equation splitted on number of one-particle equations for particles with weak interaction.

Interactions consisting the Breit Hamiltonian enter in it in additive way, and, consequently, results obtained for every interaction separately give full result by summation of each other. As next step in investigations of quantum hydrodynamics developed on base of the Breit Hamiltonian, in this work the current-current interaction and the relativistic correction to kinetic energy of particle are considered. It gives opportunity to use derived framework for wide class of physical systems, considering, for example, that solar plasma is not ultrarelativistic $\left(T \ll m c^{2}\right)$. The current-current interaction plays significant role at investigations of charged beams.

Application of the Wigner kinetics spreads with increases of interest to quantum plasmas in the last years [50], 62]-69], including generalizations for spinning particles. In most cases authors use the Wigner distribution function defined in terms of oneparticle wave functions of independent particles obeying the one-particle Schrödinger equations. As the result it gives closed mathematical apparatus looking like quantum Vlasov equation in the self-consistent field approximation. However, the general Wigner distribution function may be used for derivation of more general chain of kinetic equation to be truncated at necessary step [23]. The Wigner kinetic technic was also applied in Refs. [70], [71, where authors consider interaction under some general condition and derive corresponding quantum hydrodynamic equations applying the principle of quantum maximum entropy to close set of equations. Some attempts to get alternative kinetic methods have been also recently performed [72]-75].

Having kinetic equation one can derive corresponding quantum hydrodynamic equations, so that obtained set does not restricted by the continuity and Euler equations (corresponding set of QHD equations for spinning particles also contains the generalized Bloch equation for the spin density evolution). It allows to find equations for the energy evolution, the pressure evolution etc. We have not tested capabilities of the Wigner kinetics, but we have not seen derivation of explicit form of the momentum and energy fluxes containing quantum Bohm potentials. Method of the many-particle QHD, which is under derivation in this paper, also allows to get equations for evolution of the energy, 
pressure, energy flux etc, but in opposite to the Wigner kinetics, it allows to obtain explicit form of the fluxes including quantum parts related to the quantum Bohm potential (see for instance [1], [2], [5], and below in this paper). Non-relativistic quantum energy evolution equation including the Bohm potential was considered in Ref. [76] to get influence of the energy evolution on the Langmuir wave dispersion.

One more method of QHD derivation has been suggested recently [77]. This method also gives full chain of hydrodynamic equations. Particularly, in Ref. [77] author discuss derivation the energy density, the positivity of the entropy production is used in the derivation and hence the viscous tensors and the heat current are obtained applying the linear irreversible thermodynamics to construct a hydrodynamic model with spin. The method suggested by T. Koide allows to obtain full chain of hydrodynamic equation for quantum mediums as we do it in the many-particle QHD. However, we do not apply concepts additional to quantum mechanics and keep to stand in terms of one concept. Nevertheless, irreversibility is a fundamental property of processes in nature, and its including enriches quantum theory.

This paper is dedicated to wider consideration of the many-particle QHD method for charged spinless particles in the semi-relativistic approximation. Comparison with the classic approach and account of the energy evolution were also performed in the paper.

This paper is organized as follows. In Section 2 we derive equations for the classic semi-relativistic hydrodynamics of plasmas. In Section 3 description of QHD method is given. In Sections 4-6 we derive continuity equation, momentum and energy balance equations correspondingly. Velocity field is introduced, and obtained equations are expressed through it in Section 7. Semi-relativistic part of the quantum Bohm potential is derived in Section 7. An explicit form of the quantum thermal current up to the semi-relativistic contribution is also obtained in Section 7. As an example, in Section 8 we discuss the longitudinal waves in semi-relativistic plasmas. Conclusion is presented in Section 9.

\section{Foundations of classical semi-relativistic hydrodynamics}

Until we analyze equation of quantum hydrodynamics, let us deduce the classical equations. It needs to be done for comparison with quantum equations obtained later. This consideration is conducted with method suggested in 1996 [78]. It was a branch of more general scheme [79]. Recent discussion of some method details can be found in Ref. [80. This method allows to obtain hydrodynamical equations from microscopic equations of dynamics for $\mathrm{N}$ particles and definition of microscopic density. Some properties of relativistic hydrodynamic was considered in terms of this method in Ref. [30].

Using Lagrange equations

$$
\frac{d}{d t} \frac{\partial L}{\partial v_{i \alpha}}=\frac{\partial L}{\partial x_{i \alpha}},
$$

we obtain classical equation of motion derived from the Darwin Lagrangian (11). From 
this we express acceleration of particle $\dot{v}_{i \alpha}$ inverting structure placed before it. It cannot be carried out exactly, but, keeping terms only to the second order of $v / c$, we obtain next result:

$$
\begin{aligned}
\dot{v}_{i \alpha}= & \frac{e_{i}}{m_{i}}\left[\left(1-\frac{v_{i}^{2}}{2 c^{2}}\right) \delta_{\alpha \beta}-\frac{1}{c^{2}} v_{i \alpha} v_{i \beta}\right]\left(E_{i \beta}^{e x t}+E_{i \beta}^{i n t}\right) \\
& +\frac{e_{i}}{m_{i} c} \varepsilon_{\alpha \beta \gamma} v_{i \beta}\left(B_{i \gamma}^{e x t}+B_{i \gamma}^{i n t}\right)+\sum_{j=1, j \neq i}^{N} \frac{e_{i} e_{j}}{2 m_{i} c^{2}} v_{j \beta} v_{j \gamma} \partial_{i \beta} G_{i j}^{\alpha \gamma} \\
& -\sum_{j=1, j \neq i}^{N} \frac{e_{i} e_{j}^{2}}{2 m_{i} m_{j} c^{2}} G_{i j}^{\alpha \beta}\left(E_{j \beta}^{e x t}+E_{j \beta}^{i n t}\right),
\end{aligned}
$$

where field $E_{\alpha}^{\text {int }}$ contains only potential part.

In correspondence with [78, [80] we define mass density in the adjacency $\Delta(\mathbf{r})$ of physically infinitesimal volume with value $\Delta$ in following way:

$$
\rho(\mathbf{r}, t)=\frac{1}{\Delta} \int_{\Delta(\mathbf{r})} d \xi \sum_{i=1}^{N} m_{i} \delta\left(\mathbf{r}+\xi-\mathbf{r}_{i}(t)\right) .
$$

For particles of one species, for instance electrons, we have simple relation between the mass density $\rho$ and the particle concentration $n: \rho(\mathbf{r}, t)=m n(\mathbf{r}, t)$.

Differentiating this expression with respect to time $t$, the continuity equation is obtained:

$$
\partial_{t} \rho(\mathbf{r}, t)+\partial_{\alpha} j^{\alpha}(\mathbf{r}, t)=0
$$

where current density is

$$
j^{\alpha}(\mathbf{r}, t)=\frac{1}{\Delta} \int_{\Delta(\mathbf{r})} d \xi \sum_{i=1}^{N} m_{i} v_{i \alpha}(t) \delta\left(\mathbf{r}+\xi-\mathbf{r}_{i}(t)\right) .
$$

Differentiating with respect to time the particle current, an equation of the current evolution is obtained. This equation corresponds to the Euler equation in hydrodynamics:

$$
\partial_{t} j^{\alpha}+\partial_{\beta} \Pi^{\alpha \beta}=e n E^{\alpha}+\frac{e}{m c} \varepsilon^{\alpha \beta \gamma} j^{\beta} B^{\gamma}+\mathcal{F}_{\alpha},
$$

where we have used $\rho(\mathbf{r}, t)=m n(\mathbf{r}, t)$. For simplicity we consider system with one kind of particles, but we keep in mind that plasmas consist of two or more kinds of particles. If we would consider several kinds of particles, we have a set of QHD equations for each kind of particles. All these equations are coupled via electromagnetic field created by particles and satisfying to the Maxwell equations. The first two terms in the right-hand side represent the Lorentz force and describe an action of an electromagnetic field on charges and currents. The first term in the right-hand side of equation (8) represents sum of the external electric field and the Coulomb interaction. The second term describes an action of the external magnetic field on the currents of system, and also the currentcurrent interaction. $\mathcal{F}_{\alpha}$ is density of force caused by the semi-relativistic effects, namely by the relativistic correction to the kinetic energy and the current-current interaction. The current-current interaction enters both the Lorentz force and $\mathcal{F}_{\alpha}$. Let us introduce 
a notion of thermal velocity $u_{i \alpha}$ particle as difference between the velocity of particle and the velocity field: $u_{i \alpha}(\mathbf{r}, t)=v_{i \alpha}(t)-v_{\alpha}(\mathbf{r}, t)$, where the velocity field $v_{\alpha}$ is defined by formula $j_{\alpha}(\mathbf{r}, t)=m n(\mathbf{r}, t) v_{\alpha}(\mathbf{r}, t)$. The current on thermal velocities equals to zero, thereby velocity field $v_{\alpha}$ is separated. The flux of particle current equals to

$$
\Pi_{\alpha \beta}(\mathbf{r}, t)=m n v_{\alpha} v_{\beta}(\mathbf{r}, t)+p_{\alpha \beta}(\mathbf{r}, t),
$$

where $p_{\alpha \beta}$ is the tensor of kinetic pressure having form

$$
p_{\alpha \beta}(\mathbf{r}, t)=\frac{1}{\Delta} \int_{\Delta(\mathbf{r})} d \xi \sum_{i=1}^{N} m_{i} u_{i \alpha} u_{i \beta} \delta\left(\mathbf{r}+\xi-\mathbf{r}_{i}(t)\right) .
$$

The electric and magnetic fields represent the sum of external fields and fields caused by charges of particles: $E^{\alpha}=E_{\text {ext }}^{\alpha}+E_{\text {int }}^{\alpha}, B^{\alpha}=B_{\text {ext }}^{\alpha}+B_{\text {int }}^{\alpha}$.

The force density $\mathcal{F}_{\alpha}$ take the form

$$
\begin{aligned}
& \mathcal{F}_{\alpha}=-\frac{e}{m c^{2}}\left[\delta_{\alpha \beta}\left(\frac{1}{2} m n v^{2}+\rho \epsilon\right)+\left(m n v_{\alpha} v_{\beta}+p_{\alpha \beta}\right)\right] E_{\beta} \\
& +\frac{e^{2}}{2 c^{2}} \int d \mathbf{r}^{\prime}\left[\partial_{\alpha} G_{\beta \gamma}\left(\mathbf{r}-\mathbf{r}^{\prime}\right)-\partial_{\beta} G_{\alpha \gamma}\left(\mathbf{r}-\mathbf{r}^{\prime}\right)\right] \pi_{\beta \gamma}\left(\mathbf{r}, \mathbf{r}^{\prime}, t\right) \\
& +\frac{e^{2}}{2 m c^{2}} n \int d \mathbf{r}^{\prime} \partial_{\gamma} G_{\alpha \beta}\left(\mathbf{r}-\mathbf{r}^{\prime}\right)\left[m n\left(\mathbf{r}^{\prime}, t\right) v_{\beta}\left(\mathbf{r}^{\prime}, t\right) v_{\gamma}\left(\mathbf{r}^{\prime}, t\right)+p_{\beta \gamma}\left(\mathbf{r}^{\prime}, t\right)\right] \\
& -\frac{e^{3}}{2 m c^{2}} n \int d \mathbf{r}^{\prime} G_{\alpha \beta}\left(\mathbf{r}-\mathbf{r}^{\prime}\right) E_{\beta}\left(\mathbf{r}^{\prime}, t\right) n\left(\mathbf{r}^{\prime}, t\right),
\end{aligned}
$$

where

$$
\begin{aligned}
& \rho \epsilon(\mathbf{r}, t)=\frac{1}{\Delta} \int_{\Delta(\mathbf{r})} d \xi \sum_{i=1}^{N} \frac{1}{2} m_{i} u_{i}^{2} \delta\left(\mathbf{r}+\xi-\mathbf{r}_{i}(t)\right), \\
& \pi_{\alpha \beta}\left(\mathbf{r}, \mathbf{r}^{\prime}, t\right)=\frac{1}{\Delta} \int_{\Delta(\mathbf{r})} d \xi \sum_{i, j=1, j \neq i}^{N} \delta\left(\mathbf{r}+\xi-\mathbf{r}_{i}\right) \delta\left(\mathbf{r}^{\prime}-\mathbf{r}_{j}\right) u_{i \alpha} u_{j \beta} .
\end{aligned}
$$

In formula (11) terms in the first line arise from the semi-relativistic correction to energy of particle. Function $\rho \epsilon(\mathbf{r}, t)$ represents non-relativistic part of kinetic energy of thermal motion, and presence of term with tensor $\pi_{\alpha \beta}\left(\mathbf{r}, \mathbf{r}^{\prime}, t\right)$ in equations associated with the fact that thermal motion of particles also causes magnetic field, which give an influence on evolution of particle current. The term in the third line appears from the current-current interaction between particles, and the term in the fourth line is caused by dependence of particle acceleration (4) from the function of current-current interaction.

Let us consider the energy balance equation in classical hydrodynamics. This equation is the fifth equation in the five momentum approximation. Energy density of system is defined by formula

$$
\begin{aligned}
\varepsilon(\mathbf{r}, t)= & \frac{1}{\Delta} \int_{\Delta(\mathbf{r})} d \xi \sum_{i=1}^{N} \delta\left(\mathbf{r}+\xi-\mathbf{r}_{i}\right)\left[\frac{1}{2} m_{i} v_{i}^{2}+\frac{3}{8 c^{2}} m_{i} v_{i}^{4}\right. \\
& \left.+\sum_{j \neq i}^{N}\left(\frac{1}{2} e_{i} e_{j} G_{i j}+\frac{e_{i} e_{j}}{4 c^{2}} v_{i \alpha} v_{j \beta} G_{i j}^{\alpha \beta}\right)\right] .
\end{aligned}
$$


The first group of terms in this formula is density of non-relativistic kinetic energy, the second group is semi-relativistic correction to it, the third and the fourth groups are densities of the Coulomb and the current-current interactions correspondingly.

Applying the procedure described above, we obtain following equation for the energy density:

$$
\begin{aligned}
& \partial_{t} \varepsilon(\mathbf{r}, t)+\partial_{\alpha} Q^{\alpha}(\mathbf{r}, t)=e n(\mathbf{r}, t) \mathbf{v}(\mathbf{r}, t) \mathbf{E}+\alpha(\mathbf{r}, t) \\
& +\frac{e^{2}}{2} n(\mathbf{r}, t) \int d \mathbf{r}^{\prime} \partial^{\alpha} G\left(\mathbf{r}-\mathbf{r}^{\prime}\right)\left(v^{\alpha}(\mathbf{r}, t)-v^{\alpha}\left(\mathbf{r}^{\prime}, t\right)\right) n\left(\mathbf{r}^{\prime}, t\right) \\
& +\frac{e^{3}}{4 m c^{2}} n E_{\alpha}(\mathbf{r}, t) \int d \mathbf{r}^{\prime} G^{\alpha \beta}\left(\mathbf{r}-\mathbf{r}^{\prime}\right) n v_{\beta}\left(\mathbf{r}^{\prime}, t\right) \\
& -\frac{e^{3}}{4 m c^{2}} n v^{\alpha}(\mathbf{r}, t) \int d \mathbf{r}^{\prime} E^{\beta}\left(\mathbf{r}^{\prime}, t\right) G^{\alpha \beta}\left(\mathbf{r}-\mathbf{r}^{\prime}\right) n\left(\mathbf{r}^{\prime}, t\right) \\
& +\frac{e^{2}}{4 c^{2}} n v^{\alpha}(\mathbf{r}, t) \int d \mathbf{r}^{\prime} \partial^{\gamma} G^{\alpha \beta}\left(\mathbf{r}-\mathbf{r}^{\prime}\right)\left(v^{\gamma}(\mathbf{r}, t)+v^{\gamma}\left(\mathbf{r}^{\prime}, t\right)\right) n v^{\beta}\left(\mathbf{r}^{\prime}, t\right),
\end{aligned}
$$

where energy density can be represented as

$$
\begin{aligned}
& \varepsilon(\mathbf{r}, t)=\frac{1}{2} m n v^{2}+\frac{e^{2}}{2} n \int d \mathbf{r}^{\prime} G\left(\mathbf{r}-\mathbf{r}^{\prime}\right) n\left(\mathbf{r}^{\prime}, t\right)+\frac{3}{8 c^{2}} m n v^{4} \\
& +\frac{e^{2}}{4 c^{2}} n v_{\alpha} \int d \mathbf{r}^{\prime} G^{\alpha \beta}\left(\mathbf{r}-\mathbf{r}^{\prime}\right) n\left(\mathbf{r}^{\prime}, t\right) v_{\beta}\left(\mathbf{r}^{\prime}, t\right)+\rho \epsilon
\end{aligned}
$$

function $\rho \epsilon$ is introduced here, which has sense of internal energy density (coinciding with it in non-relativistic theory; in semi-relativistic theory in $\rho \epsilon$ terms combined with velocity field and thermal velocities appear):

$$
\begin{aligned}
& \rho \epsilon(\mathbf{r}, t)=\frac{1}{\Delta} \int_{\Delta(\mathbf{r})} d \xi \sum_{i=1}^{N} \delta\left(\mathbf{r}+\xi-\mathbf{r}_{i}(t)\right)\left[\frac{1}{2} m_{i} u_{i}^{2}\right. \\
& +\frac{3 m_{i}}{8 c^{2}}\left\{4\left(\mathbf{v}(\mathbf{r}, t) \mathbf{u}_{i}\right)^{2}+u_{i}^{4}+2 v^{2}(\mathbf{r}, t) u_{i}^{2}+4\left(\mathbf{v}(\mathbf{r}, t) \mathbf{u}_{i}\right) u_{i}^{2}\right\} \\
& \left.+\sum_{j \neq i}^{N} \frac{e_{i} e_{j}}{4 c^{2}} G_{i j}^{\alpha \beta}\left(v_{\alpha}(\mathbf{r}, t) u_{j \beta}+u_{i \alpha} v_{\beta}\left(\mathbf{r}^{\prime}, t\right)+u_{i \alpha} u_{j \beta}\right)\right] .
\end{aligned}
$$

In formula (17) terms caused by semi-relativistic part of the Lagrangian (1) are seen again. Terms in the second line appear from relativistic correction to kinetic energy, in the third line - from the current-current interaction.

Function of energy current $Q^{\alpha}(\mathbf{r}, t)$, which some times is called the energy flux, can be presented as a sum of three terms:

$$
Q^{\alpha}(\mathbf{r}, t)=v^{\alpha} \varepsilon+v^{\beta} p^{\alpha \beta}+q^{\alpha},
$$

where the thermal energy current $q^{\alpha}(\mathbf{r}, t)$ equals to

$$
\begin{aligned}
& q^{\alpha}(\mathbf{r}, t)=\frac{1}{\Delta} \int_{\Delta(\mathbf{r})} d \xi \sum_{i=1}^{N} \delta\left(\mathbf{r}+\xi-\mathbf{r}_{i}(t)\right)\left[\frac{1}{2} m u_{i \alpha} u_{i}^{2}+\sum_{j \neq i}^{N} \frac{1}{2} u_{i \alpha} e_{i} e_{j} G_{i j}\right. \\
& +\frac{3 m}{8 c^{2}} u_{i \alpha}\left\{4 v^{2}(\mathbf{r}, t) v_{\beta}(\mathbf{r}, t) u_{i \beta}+4\left(\mathbf{v}(\mathbf{r}, t) \mathbf{u}_{i}\right)^{2}\right. \\
& \left.+u_{i}^{4}+2 v^{2}(\mathbf{r}, t) u_{i}^{2}+4\left(\mathbf{v}(\mathbf{r}, t) \mathbf{u}_{i}\right) u_{i}^{2}\right\}
\end{aligned}
$$


Balance equations in semi-relativistic quantum hydrodynamics

$$
\left.+\sum_{j \neq i}^{N} u_{i \alpha}\left(v_{\beta}(\mathbf{r}, t) v_{\gamma}\left(\mathbf{r}^{\prime}, t\right)+v_{\beta}(\mathbf{r}, t) u_{j \gamma}+u_{i \beta} v_{\gamma}\left(\mathbf{r}^{\prime}, t\right)+u_{i \beta} u_{j \gamma}\right) G_{i j}^{\beta \gamma}\right] .
$$

Thermal work density $\alpha(\mathbf{r}, t)$ (which is part of work density producing by particles in thermal motion) in the energy balance equation (15) is defined in the next way:

$$
\begin{aligned}
& \alpha(\mathbf{r}, t)=\frac{1}{\Delta} \int_{\Delta(\mathbf{r})} d \xi \sum_{i, j=1, i \neq j}^{N} \delta\left(\mathbf{r}+\xi-\mathbf{r}_{i}(t)\right)\left[-\frac{e^{2}}{2}\left(u_{i \alpha}+u_{j \alpha}\right) \partial_{i \alpha} G_{i j}\right. \\
& +\frac{e^{2}}{4 c^{2}} \partial_{i \gamma} G_{i j}^{\alpha \beta}\left[\left(v_{\gamma}(\mathbf{r}, t)+v_{\gamma}\left(\mathbf{r}^{\prime}, t\right)+u_{i \gamma}+u_{j \gamma}\right)\left(v_{\alpha}(\mathbf{r}, t)+u_{i \alpha}\right) \times\right. \\
& \left.\times\left(v_{\beta}\left(\mathbf{r}^{\prime}, t\right)+u_{j \beta}\right)-\left(v_{\gamma}(\mathbf{r}, t)+v_{\gamma}\left(\mathbf{r}^{\prime}, t\right)\right) v_{\alpha}(\mathbf{r}, t) v_{\beta}\left(\mathbf{r}^{\prime}, t\right)\right] \\
& \left.+\frac{e^{3}}{4 m c^{2}} G_{i j}^{\alpha \beta}\left(E_{i \alpha} u_{j \beta}-E_{j \beta} u_{i \alpha}\right)\right] .
\end{aligned}
$$

The thermal energy current $q^{\alpha}(\mathbf{r}, t)$ and the thermal work density $\alpha(\mathbf{r}, t)$, unlike the non-relativistic case, include the velocity field $v^{\alpha}(\mathbf{r}, t)$.

Thus, equations of the five-moment approximation in semi-relativistic hydrodynamics were obtained. With method described above this equations were obtained for the first time.

\section{Quantum hydrodynamics. Formulation of problem in semi-relativistic approximation}

Let us consider a quantum mechanical system of $\mathrm{N}$ charged particles, interacting by the Coulomb and the current-current interactions, placed into an external electromagnetic

field. Derivation of quantum hydrodynamical equations is carried out by the method described in Refs. [1], [2], [3]. Microscopic mass density is defined by formula

$$
\rho(\mathbf{r}, t)=\int d R \sum_{i=1}^{N} m_{i} \delta\left(\mathbf{r}-\mathbf{r}_{i}\right) \psi^{*}(R, t) \psi(R, t),
$$

where $R=\left(\mathbf{r}_{1}, \ldots, \mathbf{r}_{N}\right), \mathbf{r}_{i}$ - coordinates of $i$-th particle,

$$
d R=\prod_{j=1}^{N} d \mathbf{r}_{j}
$$

$d R$ is an element of volume in $3 \mathrm{~N}$ dimensional configuration space, $d \mathbf{r}_{j}$ is the element of volume in three dimensional space of radius-vector $\mathbf{r}_{j}$. Number density and charge density are defined in the same way. Formula (21) appears as the quantum mechanical averaging [81] of the mass density operator giving microscopic quantum observable suitable for the collective effect description.

The Hamiltonian of system has form $\left(\hat{H}_{0}\right.$ is non-relativistic part of the Hamiltonian, $\hat{H}_{r}$ is semi-relativistic part):

$$
\begin{aligned}
& \hat{H}=\hat{H}_{0}+\hat{H}_{r}, \\
& \hat{H}_{0}=\sum_{i=1}^{N}\left(\frac{\mathbf{D}_{i}^{2}}{2 m_{i}}+e_{i} \varphi_{i}\right)+\frac{1}{2} \sum_{i, j=1, i \neq j}^{N} e_{i} e_{j} G_{i j},
\end{aligned}
$$


Balance equations in semi-relativistic quantum hydrodynamics

$$
\hat{H}_{r}=-\sum_{i=1}^{N} \frac{\mathbf{D}_{i}^{4}}{8 m_{i}^{3} c^{2}}-\sum_{i, j=1, i \neq j}^{N} \frac{e_{i} e_{j}}{4 m_{i} m_{j} c^{2}} G_{i j}^{\alpha \beta} D_{i}^{\alpha} D_{j}^{\beta},
$$

where $D_{i}^{\alpha}=(\hbar / i) \partial_{i}^{\alpha}-\left(e_{i} / c\right) A_{i}^{\alpha}, \partial_{i}^{\alpha}$ is derivative over coordinates of $i$-th particle, functions $\varphi_{i}=\varphi_{i}\left(\mathbf{r}_{i}, t\right), A_{i}^{\alpha}=A_{i}^{\alpha}\left(\mathbf{r}_{i}, t\right)$ are the potentials of external electromagnetic field, $\mathbf{r}_{i j}=\mathbf{r}_{i}-\mathbf{r}_{j} . G_{i j}, G_{i j}^{\alpha \beta}$ are the Green functions of the Coulomb and the currentcurrent interactions respectively, defining in the next way:

$$
G_{i j}=\frac{1}{r_{i j}}, G_{i j}^{\alpha \beta}=\frac{\delta^{\alpha \beta}}{r_{i j}}+\frac{x_{i j}^{\alpha} x_{i j}^{\beta}}{r_{i j}^{3}} .
$$

Previously, in section 2, with method of differentiation the equations (66)-(20) were derived for classical case. In sections 4 -7 equations of quantum hydrodynamics is derived.

\section{Continuity equation}

Differentiating mass density (21) with respect to time and using the Schrödinger equation with the Hamiltonian (23), the continuity equation is obtained:

$$
\partial_{t} \rho+\partial_{\alpha} j^{\alpha}=0
$$

where the quantum particle current $j^{\alpha}$ has form

$$
\begin{aligned}
j^{\alpha}(\mathbf{r}, t)= & \int d R \sum_{i=1}^{N} \delta\left(\mathbf{r}-\mathbf{r}_{i}\right)\left[\frac{1}{2} \psi^{*} D_{i}^{\alpha} \psi\right. \\
& -\frac{1}{8 m_{i}^{2} c^{2}}\left(\psi^{*} D_{i}^{\alpha} D_{i}^{2} \psi+D_{i}^{\alpha} \psi D_{i}^{* 2} \psi^{*}\right) \\
& \left.-\sum_{j=1, j \neq i}^{N} \frac{e_{i} e_{j}}{4 m_{j} c^{2}} G_{i j}^{\alpha \beta} \psi^{*} D_{j}^{\beta} \psi+c . c .\right] .
\end{aligned}
$$

Form of the continuity equation in comparison with the non-relativistic case does not change, but expression for the current obtains corresponding corrections.

\section{Euler equation}

In the same way, differentiating with respect to time expression for current (28), we obtain the quantum equation of particle current evolution (the Euler equation):

$$
\partial_{t} j^{\alpha}+\partial_{\beta} \Pi^{\alpha \beta}=e n E_{\text {ext }}^{\alpha}+\frac{e}{m c} \varepsilon^{\alpha \beta \gamma} j^{\beta} B_{\text {ext }}^{\gamma}+\mathcal{F}^{\alpha} .
$$

General form of this equation coincides with classical analog (8). However, functions

$\Pi^{\alpha \beta}$ and $\mathcal{F}_{\alpha}$ contain quantum contribution. The quantum flux of particle current $\Pi^{\alpha \beta}(\mathbf{r}, t)$ equals to

$$
\begin{aligned}
& \Pi^{\alpha \beta}=\int d R \sum_{i=1}^{N} \delta\left(\mathbf{r}-\mathbf{r}_{i}\right)\left[\frac{1}{4 m_{i}}\left(\psi^{*} D_{i}^{\beta} D_{i}^{\alpha} \psi+D_{i}^{* \beta} \psi^{*} D_{i}^{\alpha} \psi\right)\right. \\
& -\frac{1}{8 m_{i}^{3} c^{2}}\left(\psi^{*} D_{i}^{\beta} D_{i}^{\alpha} D_{i}^{2} \psi+D_{i}^{* \beta} \psi^{*} D_{i}^{\alpha} D_{i}^{2} \psi+D_{i}^{* \alpha} \psi^{*} D_{i}^{\beta} D_{i}^{2} \psi\right.
\end{aligned}
$$


Balance equations in semi-relativistic quantum hydrodynamics

$$
\begin{aligned}
& \left.+D_{i}^{* 2} \psi^{*} D_{i}^{\beta} D_{i}^{\alpha} \psi\right)-\sum_{j=1, j \neq i}^{N} \frac{e_{i} e_{j}}{8 m_{i} m_{j} c^{2}}\left[G_{i j}^{\beta \gamma}\left(\psi^{*} D_{j}^{\gamma} D_{i}^{\alpha} \psi+D_{j}^{* \gamma} \psi^{*} D_{i}^{\alpha} \psi\right)\right. \\
& \left.\left.+G_{i j}^{\alpha \gamma}\left(\psi^{*} D_{j}^{\gamma} D_{i}^{\beta} \psi+D_{j}^{* \gamma} \psi^{*} D_{i}^{\beta} \psi\right)\right]+c . c .\right] .
\end{aligned}
$$

In non-relativistic limit the flux of particle current coincides with the momentum current, but in semi-relativistic and full relativistic cases this is no longer true [30].

As in the case of the current, the tensor of flux of the particle current contains corrections appearing in the semi-relativistic approximation. It is possible to show that this tensor is symmetrical: $\Pi^{\alpha \beta}(\mathbf{r}, t)=\Pi^{\beta \alpha}(\mathbf{r}, t)$.

Force density $\mathcal{F}_{\alpha}(\mathbf{r}, t)$ can be represented as sum of three terms, which reflects contribution from various types of interaction:

$$
\mathcal{F}^{\alpha}(\mathbf{r}, t)=\mathcal{F}_{c l}^{\alpha}(\mathbf{r}, t)+\mathcal{F}_{r}^{\alpha}(\mathbf{r}, t)+\mathcal{F}_{c u r}^{\alpha}(\mathbf{r}, t),
$$

where

$$
\mathcal{F}_{c l}^{\alpha}=-\int d R \sum_{i, j=1, i \neq j}^{N} \delta\left(\mathbf{r}-\mathbf{r}_{i}\right) e_{i} e_{j} \partial_{i}^{\alpha} G_{i j} \psi^{*} \psi,
$$

describes contribution of the Coulomb interaction in the force density,

$$
\begin{aligned}
& \mathcal{F}_{r}^{\alpha}=-\int d R \sum_{i=1}^{N} \delta\left(\mathbf{r}-\mathbf{r}_{i}\right) \frac{e_{i}}{4 m_{i}^{2} c^{2}}\left[E_{i}^{\alpha}\left(\psi^{*} D_{i}^{2} \psi+c . c .\right)\right. \\
& \left.+E_{i}^{\beta}\left(\psi^{*} D_{i}^{\beta} D_{i}^{\alpha} \psi+D_{i}^{* \beta} \psi^{*} D_{i}^{\alpha} \psi+c . c .\right)-\hbar^{2} \partial_{i}^{\beta}\left(\partial_{i}^{\alpha} E_{i}^{\beta} \psi^{*} \psi\right)\right]
\end{aligned}
$$

is part of force density associated with the relativistic correction to kinetic energy. This quantity consists from three groups of terms. First of them is proportional to kinetic energy density $\mathcal{F}_{r, \varepsilon}^{\alpha}=-\frac{e}{m c^{2}} E^{\alpha} \varepsilon$, second is proportional to the flux of particle current $\mathcal{F}_{r, \Pi}^{\alpha}=-\frac{e}{m c^{2}} E^{\beta} \Pi^{\alpha \beta}$, third gives new purely quantum term in the Euler equation. It is proportional to a divergence of tensor, which consists from product of number density on spatial derivative of electric field $\mathcal{F}_{r, \hbar}^{\alpha}=\frac{e \hbar^{2}}{4 m^{2} c^{2}} \partial^{\beta}\left(n \partial^{\alpha} E^{\beta}\right)$.

Third term in equation (31) has form

$$
\begin{aligned}
& \mathcal{F}_{c u r}^{\alpha}=\int d R \sum_{i, j=1, i \neq j}^{N} \delta\left(\mathbf{r}-\mathbf{r}_{i}\right)\left[-\frac{e_{i} e_{j}^{2}}{2 m_{j} c^{2}} G_{i j}^{\alpha \beta} E_{j}^{\beta} \psi^{*} \psi\right. \\
& +\frac{e_{i} e_{j}}{8 m_{i} m_{j} c^{2}}\left(\partial_{i}^{\alpha} G_{i j}^{\beta \gamma}-\partial_{i}^{\beta} G_{i j}^{\alpha \gamma}\right)\left(\psi^{*} D_{i}^{\beta} D_{j}^{\gamma} \psi+D_{i}^{* \beta} \psi^{*} D_{j}^{\gamma} \psi+c . c .\right) \\
& +\frac{e_{i} e_{j}}{8 m_{j}^{2} c^{2}} \partial_{i}^{\gamma} G_{i j}^{\alpha \beta}\left(\psi^{*} D_{j}^{\beta} D_{j}^{\gamma} \psi+D_{j}^{* \beta} \psi^{*} D_{j}^{\gamma} \psi+c . c .\right) \\
& \left.-\frac{e_{i} e_{j} \hbar^{2}}{8 m_{i} m_{j} c^{2}} \partial_{i}^{\alpha} G_{i j}^{\beta \gamma} \partial_{i}^{\beta} \partial_{j}^{\gamma}\left(\psi^{*} \psi\right)\right]
\end{aligned}
$$

and represents part of field density associated with the current-current interaction between particles. It consists from four groups of terms. The first group corresponds to existing in classical theory term arising from explicit dependence of particle momentum from velocities of other particles $\left(\mathbf{p}_{i} \neq m_{i} \mathbf{v}_{i}\right)$. The second group being considered in the self-consistent field approximation, with dropping thermal contribution as well, leads 
to magnetic part of the Lorentz force $e\left[\mathbf{j}, \mathbf{B}^{\text {int }}\right] /(m c)$ caused by the current-current interaction (we show it explicitly in the Appendix). The third group is proportional to the flux of particle current and corresponds to the classical expression (see the third line in formula (11). The fourth group of terms is totally quantum, it has complex structure and, in particular, is proportional to second derivative of two-particle concentration (36).

Full electric field acting on $i$-th particle is defined in next way:

$$
E_{i \alpha}=E_{i, e x t}^{\alpha}-\sum_{j=1, j \neq i}^{N} e_{j} \partial_{i}^{\alpha} G_{i j} .
$$

The Euler equation, unlike the continuity equation, interaction enters in explicit form. In case of system with the Coulomb interaction obtained equation coincides by form with classical equation, however, it contains quantum correlations caused by exchange interactions. Quantum character shows in the presence of additional quantum pressure called the quantum Bohm potential.

At account of the semi-relativistic corrections, essentially new terms appear in the Euler equation. Two group of terms appear in all: first associated with the relativistic correction to kinetic energy, second - with the current-current interaction. Part of these terms is classical, i. e. appears at constructing of classical hydrodynamics based on the Darwin Lagrangian (but we should note that the flux of particle current and the energy density contain quantum parts, which is absent in the classical theory). Essentially new terms are the quantum terms explicitly proportional to $\hbar$.

Deriving equations of quantum hydrodynamics we start from definition of number (or mass) density, its differentiation gives continuity equation. In this equation expression for current density through wave function appears. In equation for current density new functions appear, for which by differentiation over time evolution equations can be derived. In this way infinite chain of equations in 3D physical space is obtained, similar to BBGKY chain in kinetics. This chain of equations is equivalent to the initial Schrödinger equation in configuration space.

Equations obtained above are written in general form. We consider these equations in self-consistent field approximation further. If we introduce two-particle concentration in the Coulomb force field (32)

$$
n_{2}\left(\mathbf{r}, \mathbf{r}^{\prime}, t\right)=\int d R \sum_{i, j=1, i \neq j}^{N} \delta\left(\mathbf{r}-\mathbf{r}_{i}\right) \delta\left(\mathbf{r}^{\prime}-\mathbf{r}_{j}\right) \psi^{*}(R, t) \psi(R, t),
$$

then in case of system consisting from one kind of particles, and also in self-consistent field approximation $\left(n_{2}\left(\mathbf{r}, \mathbf{r}^{\prime}, t\right)=n(\mathbf{r}, t) n\left(\mathbf{r}^{\prime}, t\right)\right)$, expression for force density of the Coulomb interaction $\mathcal{F}_{c l}^{\alpha}$ take form

$$
\mathcal{F}_{c l}^{\alpha}(\mathbf{r}, t)=-e^{2} n(\mathbf{r}, t) \partial^{\alpha} \int G\left(\mathbf{r}-\mathbf{r}^{\prime}\right) n\left(\mathbf{r}^{\prime}, t\right) d \mathbf{r}^{\prime} .
$$

Further, after introduction of velocity field in section 7, all quantities in obtained equations are written in the self-consistent field approximation. 


\section{Energy balance equation}

Investigating hydrodynamical equations five-moment approximation is considered usually, consisting of continuity equation, momentum balance equation and energy balance equation. Continuity equation and momentum balance equation were obtained above. Now we proceed to derivation of quantum energy balance equation. Energy density for considered system of particles is defined in next way:

$$
\begin{aligned}
& \varepsilon(\mathbf{r}, t)=\int d R \sum_{i=1}^{N} \delta\left(\mathbf{r}-\mathbf{r}_{i}\right)\left[\left(\frac{1}{4 m_{i}} \psi^{*} D_{i}^{2} \psi-\frac{1}{16 m_{i}^{3} c^{2}} \psi^{*} D_{i}^{4} \psi+c . c .\right)\right. \\
& \left.+\sum_{j=1, j \neq i}^{N}\left(\frac{1}{2} e_{i} e_{j} G_{i j} \psi^{*} \psi-\frac{e_{i} e_{j}}{8 m_{i} m_{j} c^{2}} G_{i j}^{\alpha \beta}\left(\psi^{*} D_{i}^{\alpha} D_{j}^{\beta} \psi+c . c .\right)\right)\right] .
\end{aligned}
$$

As in the case of mass current density (28), differentiating this expression over time and using Schrödinger equation we obtain energy balance equation:

$$
\partial_{t} \varepsilon(\mathbf{r}, t)+\partial_{\alpha} Q^{\alpha}(\mathbf{r}, t)=j_{e}^{\alpha}(\mathbf{r}, t) E_{\alpha}(\mathbf{r}, t)+\mathcal{A}(\mathbf{r}, t),
$$

where $Q^{\alpha}(\mathbf{r}, t)$ is vector of energy current density, $\mathcal{A}(\mathbf{r}, t)$ is work density. Vector of energy current density has complex structure. We divide it on three parts

$$
Q^{\alpha}(\mathbf{r}, t)=Q_{0}^{\alpha}(\mathbf{r}, t)+Q_{\text {coul }}^{\alpha}(\mathbf{r}, t)+Q_{r, \text { cur }}^{\alpha}(\mathbf{r}, t),
$$

and consider them separately. First term in (40) is non-relativistic kinetic energy current density (see [1]) and has form

$$
Q_{0}^{\alpha}=\int d R \sum_{i=1}^{N} \delta\left(\mathbf{r}-\mathbf{r}_{i}\right) \frac{1}{8 m_{i}^{2}}\left(\psi^{*} D_{i}^{\alpha} D_{i}^{2} \psi+D_{i}^{* \alpha} \psi^{*} D_{i}^{2} \psi+\text { c. c. }\right) .
$$

Energy current of the Coulomb interaction is represented by second term in formula (40):

$$
\begin{aligned}
& Q_{\text {coul }}^{\alpha}=\int d R \sum_{i, j=1, i \neq j}^{N} \delta\left(\mathbf{r}-\mathbf{r}_{i}\right) e_{i} e_{j} G_{i j}\left[\frac{1}{4 m_{i}}\left(\psi^{*} D_{i}^{\alpha} \psi+c . c .\right)\right. \\
& -\frac{1}{16 m_{i}^{3} c^{2}}\left(\psi^{*} D_{i}^{\alpha} D_{i}^{2} \psi+D_{i}^{* \alpha} \psi^{*} D_{i}^{2} \psi+c . c .\right) \\
& \left.-\sum_{k=1, k \neq j}^{N} \frac{e_{i} e_{k}}{8 m_{i} m_{k} c^{2}} G_{i k}^{\alpha \beta}\left(\psi^{*} D_{k}^{\beta} \psi+\text { c. c. }\right)\right]
\end{aligned}
$$

where expression in square brackets is analogous by its structure to the current $j^{\alpha}(28)$ and consists from three parts. First part corresponds to result obtained earlier from non-relativistic theory [1, second and third parts are caused by contribution from semirelativistic effects (the addition to kinetic energy and the current-current interaction, correspondingly).

Part of energy current $Q_{r, c u r}^{\alpha}$ arising from semi-relativistic corrections in the Hamiltonian (25) is defined by expression

$$
Q_{r, \text { cur }}^{\alpha}=-\int d R \sum_{i=1}^{N} \delta\left(\mathbf{r}-\mathbf{r}_{i}\right)\left[\frac { 1 } { 1 6 m _ { i } ^ { 4 } c ^ { 2 } } \left(\psi^{*} D_{i}^{\alpha} D_{i}^{4} \psi\right.\right.
$$


Balance equations in semi-relativistic quantum hydrodynamics

$$
\begin{aligned}
& \left.+D_{i}^{* \alpha} \psi^{*} D_{i}^{4} \psi+D_{i}^{* 2} \psi^{*} D_{i}^{\alpha} D_{i}^{2} \psi+c . c .\right) \\
& +\sum_{j=1, j \neq i}^{N} \frac{e_{i} e_{j}}{16 m_{i}^{2} m_{j} c^{2}}\left\{G_{i j}^{\alpha \beta}\left(\psi^{*} D_{j}^{\beta} D_{i}^{2} \psi+D_{j}^{* \beta} \psi^{*} D_{i}^{2} \psi+c . c .\right)\right. \\
& \left.\left.+G_{i j}^{\beta \gamma}\left(\psi^{*} D_{i}^{\alpha} D_{i}^{\beta} D_{j}^{\gamma} \psi+D_{i}^{* \alpha} \psi^{*} D_{i}^{\beta} D_{j}^{\gamma} \psi+c . c .\right)\right\}\right] .
\end{aligned}
$$

Terms in the first two lines arise from the semi-relativistic correction to kinetic energy, in the last two lines - from the current-current interaction between particles.

Expression for work density $\mathcal{A}(\mathbf{r}, t)$ can be also divided in three parts:

$$
\mathcal{A}(\mathbf{r}, t)=\mathcal{A}^{c l}(\mathbf{r}, t)+\mathcal{A}^{r}(\mathbf{r}, t)+\mathcal{A}^{c u r}(\mathbf{r}, t),
$$

where

$$
\begin{aligned}
& \mathcal{A}^{c l}=\int d R \sum_{i, j=1, j \neq i}^{N} \delta\left(\mathbf{r}-\mathbf{r}_{i}\right) e_{i} e_{j} \partial_{i}^{\alpha} G_{i j}\left[\frac{1}{4 m_{i}}\left(\psi^{*} D_{i}^{\alpha} \psi+\text { c. c. }\right)\right. \\
& -\frac{1}{4 m_{j}}\left(\psi^{*} D_{j}^{\alpha} \psi+\text { c. c. }\right)-\frac{1}{16 m_{i}^{3} c^{2}}\left(\psi^{*} D_{i}^{\alpha} D_{i}^{2} \psi+D_{i}^{* \alpha} \psi^{*} D_{i}^{2} \psi\right. \\
& + \text { c. c. })+\frac{1}{16 m_{j}^{3} c^{2}}\left(\psi^{*} D_{j}^{\alpha} D_{j}^{2} \psi+D_{j}^{* \alpha} \psi^{*} D_{j}^{2} \psi+c . c .\right) \\
& -\sum_{k=1, k \neq i}^{N} \frac{e_{i} e_{k}}{8 m_{i} m_{k} c^{2}} G_{i k}^{\alpha \beta}\left(\psi^{*} D_{k}^{\beta} \psi+\text { c. c. }\right) \\
& \left.+\sum_{k=1, k \neq j}^{N} \frac{e_{j} e_{k}}{8 m_{j} m_{k} c^{2}} G_{j k}^{\alpha \beta}\left(\psi^{*} D_{k}^{\beta} \psi+\text { c. c. }\right)\right],
\end{aligned}
$$

$\mathcal{A}^{\text {cl }}$ is the Coulomb part of work density (it is seen from the definition of mass current density (28) and formula $j^{\alpha}(\mathbf{r}, t)=\int d R \sum_{i=1}^{N} \delta\left(\mathbf{r}-\mathbf{r}_{i}\right) \psi^{*} \psi m_{i} v_{i \alpha}$, from which it is seen that

$$
\begin{aligned}
v_{i \alpha}= & \frac{1}{2 m_{i}} \frac{D_{i}^{\alpha} \psi}{\psi}-\frac{1}{8 m_{i}^{3} c^{2}} \frac{1}{\psi^{*} \psi}\left(\psi^{*} D_{i}^{\alpha} D_{i}^{2} \psi+D_{i}^{* \alpha} \psi^{*} D_{i}^{2} \psi\right) \\
& -\sum_{j=1, j \neq i}^{N} \frac{e_{i} e_{j}}{4 m_{i} m_{j} c^{2}} G_{i j}^{\alpha \beta} \frac{D_{j}^{\beta} \psi}{\psi}+c . c .,
\end{aligned}
$$

after this it is easy to see correspondence between formula for $\mathcal{A}^{c l}$ and corresponding part of classical energy balance equation (15)). Work density $\mathcal{A}^{r}$ associated with the relativistic correction to kinetic energy looks in the following way:

$$
\begin{aligned}
& \mathcal{A}^{r}=\int d R \sum_{i=1}^{N} \delta\left(\mathbf{r}-\mathbf{r}_{i}\right) \frac{e_{i}}{8 m_{i}^{3} c^{2}}\left[\hbar^{2} \partial_{i}^{\alpha}\left[\partial_{i}^{\beta} E_{i}^{\beta}\left(\psi^{*} D_{i}^{\alpha} \psi+c . c .\right)\right]\right. \\
& +\hbar^{2} \Delta_{i} E_{i}^{\alpha}\left(\psi^{*} D_{i}^{\alpha} \psi+c . c .\right)+\hbar^{2} E_{i}^{\alpha} \partial_{i}^{\alpha} \partial_{i}^{\beta}\left(\psi^{*} D_{i}^{\beta} \psi+\text { c. c. }\right) \\
& \left.-2 \frac{\hbar}{i} \partial_{i}^{\alpha} E_{i}^{\beta}\left(\psi^{*} D_{i}^{\alpha} D_{i}^{\beta} \psi-\text { c. c. }\right)\right] .
\end{aligned}
$$

This function is proportional to square of Planck constant $\hbar$ (with respect to fact that expression in the last line also gives square of $\hbar$ at expansion). Terms in the first two lines are proportional to derivative of electric field and current density, and with respect 
to this fact this expression (in the case of system with one kind of particles) can be written in the following form:

$$
\begin{aligned}
& \mathcal{A}^{r}=\frac{e \hbar^{2}}{4 m^{3} c^{2}}\left[\partial_{\alpha}\left(\partial_{\beta} E_{\beta} j^{\alpha}\right)+\Delta E_{\alpha} j^{\alpha}+E_{\alpha} \partial_{\alpha}\left(\partial_{\beta} j^{\beta}\right)\right. \\
& \left.+\partial_{\alpha} E_{\beta} \int d R \sum_{i=1}^{N} \delta\left(\mathbf{r}-\mathbf{r}_{i}\right) \frac{i}{\hbar}\left(\psi^{*} D_{i}^{\alpha} D_{i}^{\beta} \psi-\text { c. c. }\right)\right] .
\end{aligned}
$$

Finally we obtain work density $\mathcal{A}^{\text {cur }}$ associated with the current-current interaction between particles:

$$
\begin{aligned}
& \mathcal{A}^{\text {cur }}=\int d R \sum_{i, j=1, j \neq i}^{N} \delta\left(\mathbf{r}-\mathbf{r}_{i}\right) \frac{e_{i} e_{j}}{8 m_{i} m_{j} c^{2}}\left[e_{i} E_{i}^{\alpha} G_{i j}^{\alpha \beta}\left(\psi^{*} D_{j}^{\beta} \psi+c . c .\right)\right. \\
& -e_{j} E_{j}^{\beta} G_{i j}^{\alpha \beta}\left(\psi^{*} D_{i}^{\alpha} \psi+c . c .\right)+\frac{2}{m_{i}} \partial_{i}^{\gamma} G_{i j}^{\alpha \beta}\left(\psi^{*} D_{i}^{\gamma} D_{i}^{\alpha} D_{j}^{\beta} \psi+c . c .\right) \\
& -\frac{1}{2 m_{i}} \partial_{i}^{\gamma} G_{i j}^{\alpha \beta}\left(\psi^{*} D_{i}^{\gamma} D_{i}^{\alpha} D_{j}^{\beta} \psi+D_{i}^{* \gamma} \psi^{*} D_{i}^{\alpha} D_{j}^{\beta} \psi+c . c .\right) \\
& +\frac{1}{2 m_{j}} \partial_{i}^{\gamma} G_{i j}^{\alpha \beta}\left(\psi^{*} D_{j}^{\gamma} D_{i}^{\alpha} D_{j}^{\beta} \psi+D_{j}^{* \gamma} \psi^{*} D_{i}^{\alpha} D_{j}^{\beta} \psi+c . c .\right) \\
& \left.+\frac{\hbar}{i m_{i}} \Delta_{i} G_{i j}^{\alpha \beta}\left(\psi^{*} D_{i}^{\alpha} D_{j}^{\beta} \psi-c . c .\right)\right] .
\end{aligned}
$$

Expression in the last line is completely quantum. Other terms correspond to the classical current-current terms in the right-hand side of energy balance equation (15), but as in the case of tensor of the flux of particle current (30) they give a quantum contribution. So we have an analog of the quantum Bohm potential.

\section{Introduction of velocity field}

\subsection{Exponential form of wave function and velocity of particle}

Basic fact is the fact that mass current density is $j_{\alpha}(\mathbf{r}, t)=m n(\mathbf{r}, t) v_{\alpha}(\mathbf{r}, t)$, i. e. velocity field $v_{\alpha}(\mathbf{r}, t)$ is defined from obtained above expressions for number density and current density.

Wave function of $\mathrm{N}$ particle system can be represented as

$$
\psi(R, t)=a(R, t) \exp (i S(R, t) / \hbar),
$$

In formula (50), which is called the Madelung decomposition, $a^{2}(R, t)$ is the probability density of detection in volume $(R, R+d R)$ of $3 \mathrm{~N}$ dimensional configuration space, and

phase of wave function $S(R, t)$ is quantity, gradient of which corresponds to probability current in non-relativistic case.

Substituting given expression (50) in the formula for the current (28) we obtain:

$$
j^{\alpha}(\mathbf{r}, t)=\int d R \sum_{i=1}^{N} \delta\left(\mathbf{r}-\mathbf{r}_{i}\right) m_{i} a^{2} v_{i}^{\alpha},
$$


where

$$
\begin{aligned}
v_{i}^{\alpha}= & \frac{s_{i}^{\alpha}}{m_{i}}-\frac{s_{i}^{\alpha} s_{i}^{2}}{2 m_{i}^{3} c^{2}}+\frac{\hbar^{2}}{2 m_{i}^{3} c^{2}}\left[s_{i}^{\alpha} a^{-1} \Delta_{i} a+\partial_{i}^{\alpha}\left(s_{i}^{\beta} \partial_{i}^{\beta} \ln a\right)\right. \\
& \left.+\frac{1}{2} \partial_{i}^{\alpha} \partial_{i}^{\beta} s_{i}^{\beta}\right]-\sum_{j=1, j \neq i}^{N} \frac{e_{i} e_{j}}{2 m_{i} m_{j} c^{2}} G_{i j}^{\alpha \beta} s_{j}^{\beta}
\end{aligned}
$$

and quantity $\mathbf{s}_{i}$ is defined as $s_{i}^{\alpha}=\partial_{i}^{\alpha} S-\frac{e_{i}}{c} A_{i}^{\alpha}$. Velocity of $i$-th particle should be divided into the velocity field and the thermal velocity of of $i$ th particle: $v_{i}^{\alpha}(R, t)=$ $v^{\alpha}(\mathbf{r}, t)+u_{i}^{\alpha}(\mathbf{r}, R, t)$. The velocity field is introduced in order that the current equals to zero on the thermal velocities:

$$
\int d R \sum_{i=1}^{N} \delta\left(\mathbf{r}-\mathbf{r}_{i}\right) m_{i} a^{2} u_{i}^{\alpha}=0 .
$$

As a result we obtain familiar expression for the mass current density (for convenience we consider system with one kind of particles, i. e. $m_{i}=m, e_{i}=e$ for each $i$ ):

$$
j^{\alpha}(\mathbf{r}, t)=m n(\mathbf{r}, t) v^{\alpha}(\mathbf{r}, t) .
$$

In terms of the velocity field, the continuity equation takes the well-known form form

$$
\partial_{t} n(\mathbf{r}, t)+\partial_{\alpha}\left[n(\mathbf{r}, t) v^{\alpha}(\mathbf{r}, t)\right]=0 .
$$

\subsection{Equation for the velocity field evolution}

Using the Madelung decomposition for wave function (50) current and energy evolution equations can be expressed through familiar hydrodynamical and thermodynamical quantities.

For system with one kind of particles the current evolution equation takes form

$$
\begin{aligned}
& m n(\mathbf{r}, t)\left(\partial_{t}+v_{\beta}(\mathbf{r}, t) \partial_{\beta}\right) v_{\alpha}(\mathbf{r}, t)+\partial_{\beta} p_{\alpha \beta}(\mathbf{r}, t)+\partial_{\beta} T_{\alpha \beta}(\mathbf{r}, t)= \\
& =e n E_{\alpha}+\frac{e}{c} n \varepsilon_{\alpha \beta \gamma} v_{\beta} B_{\gamma}+\mathcal{F}_{\alpha}^{c l}+\mathcal{F}_{\alpha}^{q},
\end{aligned}
$$

where $p_{\alpha \beta}(\mathbf{r}, t)$ is the kinetic pressure tensor, $T_{\alpha \beta}(\mathbf{r}, t)$ is the quantum addition to the thermal pressure (the quantum Bohm potential), the electric field $\mathbf{E}$ is the sum of the external field and the field associated with the interaction between particles: $E_{\alpha}(\mathbf{r}, t)=$ $E_{\alpha}^{e x t}(\mathbf{r}, t)+E_{\alpha}^{i n t}(\mathbf{r}, t)$, and similarly for the magnetic field: $B_{\alpha}(\mathbf{r}, t)=B_{\alpha}^{e x t}(\mathbf{r}, t)+B_{\alpha}^{i n t}(\mathbf{r}, t)$.

Function $\mathcal{F}_{\alpha}^{c l}$ is the classical semi-relativistic part of force density (see (11); one needs to account the fact that functions $p_{\alpha \beta}(\mathbf{r}, t)$ and $\rho \epsilon(\mathbf{r}, t)$ contain quantum part, for example, instead of $p_{\alpha \beta}$ it should be written $p_{\alpha \beta}+T_{\alpha \beta}$ ), and $\mathcal{F}_{\alpha}^{q}$ is a specific part of the force density appearing in the QHD:

$$
\begin{aligned}
& \mathcal{F}_{\alpha}^{q}=\frac{e \hbar^{2}}{4 m^{2} c^{2}} \partial_{\beta}\left(\partial_{\alpha} E_{\beta} \cdot n\right) \\
& -\frac{e^{2} \hbar^{2}}{8 m^{2} c^{2}} \partial_{\beta} n(\mathbf{r}, t) \int d \mathbf{r}^{\prime} \partial_{\alpha} G_{\beta \gamma}\left(\mathbf{r}-\mathbf{r}^{\prime}\right) \partial_{\gamma}^{\prime} n\left(\mathbf{r}^{\prime}, t\right) .
\end{aligned}
$$

Fields $\mathbf{E}^{\text {int }}, \mathbf{B}^{\text {int }}$ associated with interaction between particles of system satisfy to following formulas:

$$
\mathbf{E}^{i n t}(\mathbf{r}, t)=-\nabla \varphi^{i n t}(\mathbf{r}, t)
$$


Balance equations in semi-relativistic quantum hydrodynamics

and

$$
\mathbf{B}^{i n t}(\mathbf{r}, t)=\operatorname{curl}^{i n t}(\mathbf{r}, t),
$$

with

$$
\varphi^{i n t}(\mathbf{r}, t)=e \int d \mathbf{r}^{\prime} G\left(\mathbf{r}-\mathbf{r}^{\prime}\right) n\left(\mathbf{r}^{\prime}, t\right)
$$

and

$$
A_{\alpha}^{i n t}(\mathbf{r}, t)=\frac{e}{2 c} \int d \mathbf{r}^{\prime} G_{\alpha \beta}\left(\mathbf{r}-\mathbf{r}^{\prime}\right) n\left(\mathbf{r}^{\prime}, t\right) v_{\beta}\left(\mathbf{r}^{\prime}, t\right) .
$$

These fields obey the quasi-static Maxwell equations:

$$
\begin{aligned}
& \nabla \mathbf{E}(\mathbf{r}, t)=4 \pi \sum_{a} e_{a} n_{a}(\mathbf{r}, t), \\
& \nabla \times \mathbf{E}(\mathbf{r}, t)=0 \\
& \nabla \mathbf{B}(\mathbf{r}, t)=0
\end{aligned}
$$

and

$$
\nabla \times \mathbf{B}(\mathbf{r}, t)=\frac{4 \pi}{c} \sum_{a} e_{a} n_{a}(\mathbf{r}, t) \mathbf{v}_{a}(\mathbf{r}, t),
$$

where $a$ stands for species of particles.

Tensor $\pi_{\alpha \beta}\left(\mathbf{r}, \mathbf{r}^{\prime}, t\right)$ entering in $\mathcal{F}_{\alpha}^{c l}$ (11) looks like

$$
\begin{aligned}
& \pi_{\alpha \beta}\left(\mathbf{r}, \mathbf{r}^{\prime}, t\right)= \\
& \quad=\int d R \sum_{i, j=1, i \neq j}^{N} \delta\left(\mathbf{r}-\mathbf{r}_{i}\right) \delta\left(\mathbf{r}^{\prime}-\mathbf{r}_{j}\right) a^{2}\left(u_{i \alpha} u_{j \beta}-\frac{\hbar^{2}}{2 m^{2}} \partial_{i \alpha} \partial_{j \beta} \ln a\right) .
\end{aligned}
$$

Tensor $T_{\alpha \beta}(\mathbf{r}, t)$ corresponding to the quantum Bohm potential has form

$$
\begin{aligned}
& T_{\alpha \beta}(\mathbf{r}, t)=\int d R \sum_{i=1}^{N} \delta\left(\mathbf{r}-\mathbf{r}_{i}\right) a^{2}\left[-\frac{\hbar^{2}}{2 m}\left(1-\frac{v_{i}^{2}}{c^{2}}\right) \partial_{i \alpha} \partial_{i \beta} \ln a\right. \\
& +\frac{\hbar^{2}}{2 m c^{2}}\left(\partial_{i \alpha} v_{i \gamma} \partial_{i \beta} v_{i \gamma}+v_{i \gamma} \partial_{i \alpha} \partial_{i \beta} v_{i \gamma}\right) \\
& +\frac{\hbar^{2}}{4 m c^{2}}\left(v_{i \alpha} \partial_{i \beta}+v_{i \beta} \partial_{i \alpha}\right)\left(\partial_{i \gamma} v_{i \gamma}+2 v_{i \gamma} \partial_{i \gamma} \ln a\right) \\
& \left.-\frac{\hbar^{4} a^{-2}}{4 m^{3} c^{2}}\left(a \partial_{i \alpha} \partial_{i \beta} \Delta_{i} a+\partial_{i \alpha} \partial_{i \beta} a \Delta_{i} a-\partial_{i \alpha} a \partial_{i \beta} \Delta_{i} a-\partial_{i \beta} a \partial_{i \alpha} \Delta_{i} a\right)\right] \\
& +\int d R \sum_{i=1, j=1, i \neq j}^{N} \delta\left(\mathbf{r}-\mathbf{r}_{i}\right) a^{2} \frac{\hbar^{2} e^{2}}{4 m^{2} c^{2}}\left(G_{i j}^{\beta \gamma} \partial_{i \alpha} \partial_{j \gamma} \ln a+G_{i j}^{\alpha \gamma} \partial_{i \beta} \partial_{j \gamma} \ln a\right) .
\end{aligned}
$$

This is a general representation of the semi-relativistic quantum Bohm potential. It is defined in terms of the amplitude $a=a(R, t)$ of many-particle wave function (50) and the quantum velocities $\mathbf{v}_{i}$ appearing via the phase of many-particle wave function (52). Usually, at applications of quantum hydrodynamics, the quantum Bohm potential is used in approximation of independent particles, see for instance Ref. [3] formulas (10)-(11), and Ref. [53] formula (17). Below we present approximate form of the semirelativistic quantum Bohm potential (67). The first term in the first group of terms in 
Balance equations in semi-relativistic quantum hydrodynamics

(67) is the non-relativistic part of the Bohm potential obtained in Ref. [1] (see formula (35)). Its approximate form is

$$
T_{n r}^{\alpha \beta}=-\frac{\hbar^{2}}{4 m} \partial^{\alpha} \partial^{\beta} n+\frac{\hbar^{2}}{4 m}\left(\frac{\partial^{\alpha} n \cdot \partial^{\beta} n}{n}\right) .
$$

We should also represent it as the density of force field

$$
F_{Q}^{\alpha}=-\partial_{\beta} T^{\alpha \beta}=\frac{\hbar^{2}}{2 m} n \partial_{\alpha} \frac{\triangle \sqrt{n}}{\sqrt{n}}
$$

to make it more recognizable.

The full semi-relativistic Bohm potential arises in approximation of independent particles as

$$
\begin{gathered}
T^{\alpha \beta}(\mathbf{r}, t)=T_{n r}^{\alpha \beta}-\frac{v^{2}}{c^{2}} T_{n r}^{\alpha \beta} \\
+\frac{\hbar^{2}}{2 m c^{2}} n\left(\partial^{\alpha} v^{\gamma} \partial^{\beta} v^{\gamma}+v^{\gamma} \partial^{\alpha} \partial^{\beta} v^{\gamma}\right)+\frac{\hbar^{2}}{4 m c^{2}} n\left(v^{\alpha} \partial^{\beta}+v^{\beta} \partial^{\alpha}\right)(\nabla \mathbf{v}) \\
+\frac{\hbar^{2}}{4 m c^{2}}\left(\partial^{\gamma} n\right)\left(v^{\alpha} \partial^{\beta} v^{\gamma}+v^{\beta} \partial^{\alpha} v^{\gamma}\right)-\frac{1}{c^{2}}\left(v^{\alpha} v^{\gamma} T_{n r}^{\beta \gamma}+v^{\beta} v^{\gamma} T_{n r}^{\alpha \gamma}\right) \\
-\frac{\hbar^{4}}{4 m^{3} c^{2}}\left(\sqrt{n} \cdot \partial^{\alpha} \partial^{\beta} \triangle \sqrt{n}+\partial^{\alpha} \partial^{\beta} \sqrt{n} \cdot \triangle \sqrt{n}\right. \\
\left.-\partial^{\alpha} \sqrt{n} \cdot \partial^{\beta} \triangle \sqrt{n}-\partial^{\beta} \sqrt{n} \cdot \partial^{\alpha} \triangle \sqrt{n}\right) .
\end{gathered}
$$

Relativistic part of this formula appears to contain two type of structures. Terms containing the velocity field $\mathbf{v}$, so they explicitly have $v^{2} / c^{2}$ to reveal their semirelativistic nature. The second type of structures is presented by the two last lines of formula (70). They have $\frac{\hbar^{2}}{m c^{2}} \triangle$ instead of $v^{2} / c^{2}$, so they give contribution in evolution of quantum plasmas even than velocity field $\mathbf{v}$ is small. Transition from formula (67) to formula (70) is a step towards closing of the QHD set.

\subsection{Energy balance equation}

Now the energy balance equation (39) should be expressed through the velocity field. After substitution of the Madelung decomposition (50) into formula for the energy density and dividing it in two parts, we obtain

$$
\varepsilon(\mathbf{r}, t)=\varepsilon_{c l}(\mathbf{r}, t)+\varepsilon_{q}(\mathbf{r}, t),
$$

where $\varepsilon_{c l}$ is classical part of energy density (see (14)):

$$
\begin{aligned}
& \varepsilon_{c l}(\mathbf{r}, t)=\int d R \sum_{i=1}^{N} \delta\left(\mathbf{r}-\mathbf{r}_{i}\right) a^{2}\left[\frac{1}{2} m v_{i}^{2}+\frac{3}{8 c^{2}} m v_{i}^{4}\right. \\
& \left.+\sum_{j=1, j \neq i}^{N}\left(\frac{1}{2} e^{2} G_{i j}+\frac{e^{2}}{4 c^{2}} G_{i j}^{\alpha \beta} v_{i}^{\alpha} v_{j}^{\beta}\right)\right]
\end{aligned}
$$


Balance equations in semi-relativistic quantum hydrodynamics

and the quantum part of written below energy density $\varepsilon_{q}$ appears as:

$$
\begin{aligned}
& \varepsilon_{q}(\mathbf{r}, t)=\frac{\hbar^{2}}{2 m} \int d R \sum_{i=1}^{N} \delta\left(\mathbf{r}-\mathbf{r}_{i}\right) a^{2}\left[-\frac{\Delta_{i} a}{a}+\frac{1}{4 c^{2}}\left[\left(\nabla_{i} \mathbf{v}_{i}\right)^{2}\right.\right. \\
& \left.+2\left(\partial_{i}^{\beta} v_{i}^{\gamma}\right)\left(\partial_{i}^{\beta} v_{i}^{\gamma}\right)+2 v_{i}^{\beta} \Delta_{i} v_{i}^{\beta}\right]+\frac{1}{c^{2}} \frac{\partial_{i}^{\beta} a}{a}\left(v_{i}^{\beta} \partial_{i}^{\alpha} v_{i}^{\alpha}+v_{i}^{\alpha} \partial_{i}^{\beta} v_{i}^{\alpha}\right) \\
& -\frac{1}{2 c^{2}} a^{-1} \Delta_{i} a v_{i}^{2}+\frac{1}{c^{2}} a^{-2} \partial_{i}^{\alpha} a \partial_{i}^{\beta} a v_{i}^{\alpha} v_{i}^{\beta}-\frac{\hbar^{2}}{4 m^{2} c^{2}} a^{-1} \Delta_{i} \Delta_{i} a \\
& \left.+\sum_{j=1, j \neq i}^{N} \frac{e^{2}}{2 m c^{2}} G_{i j}^{\alpha \beta} a^{-1} \partial_{i}^{\alpha} \partial_{j}^{\beta} a\right] .
\end{aligned}
$$

First term in formula (73) has non-relativistic quantum origin. Other terms are associated with semi-relativistic corrections, that is seen from presence of the factor $1 / c^{2}$ before them.

Separating classical current (i. e. not proportional to the thermal velocities) and the Coulomb parts, function $\varepsilon(\mathbf{r}, t)$ can be represented in following form:

$$
\begin{aligned}
\varepsilon(\mathbf{r}, t)= & \frac{1}{2} m n v^{2}+\frac{3}{8 c^{2}} m n v^{4}+\frac{e^{2}}{2} n \int d \mathbf{r}^{\prime} G\left(\mathbf{r}-\mathbf{r}^{\prime}\right) n\left(\mathbf{r}^{\prime}, t\right) \\
& +\frac{e^{2}}{4 c^{2}} n v^{\alpha} \int d \mathbf{r}^{\prime} G^{\alpha \beta}\left(\mathbf{r}-\mathbf{r}^{\prime}\right) n\left(\mathbf{r}^{\prime}, t\right) v^{\beta}\left(\mathbf{r}^{\prime}, t\right)+\rho \epsilon,
\end{aligned}
$$

where $\rho \epsilon(\mathbf{r}, t)$ may be called thermal energy density. This function is energy density without terms containing the velocity field only (i. e. without terms containing thermal velocities) in classical part of energy density $\varepsilon_{c l}$, and also the term, which is energy of the Coulomb interaction.

The energy current can be represented as follows:

$$
Q^{\alpha}=v^{\alpha} \varepsilon+v^{\beta}\left(p^{\alpha \beta}+T^{\alpha \beta}\right)+q^{\alpha} .
$$

The thermal current $q^{\alpha}$ entering in the last formula has rather complicated form. So, we represent it as a sum of several terms:

$$
\begin{aligned}
q^{\alpha}= & \int d R \sum_{i=1}^{N} \delta\left(\mathbf{r}-\mathbf{r}_{i}\right) a^{2}\left[u_{i}^{\alpha} \tilde{\rho} \epsilon_{i}+u_{i}^{\beta} \tilde{T}_{i}^{\alpha \beta}\right. \\
& \left.-\frac{\hbar^{2}}{2 m} a^{-1} \partial_{i \beta} a \partial_{i \alpha} v_{i \beta}-\frac{\hbar^{2}}{4 m} \partial_{i \alpha} \partial_{i \beta} v_{i \beta}\right] \\
& +q_{0}^{\alpha}+q_{I}^{\alpha}+q_{I I}^{\alpha}+q_{I I I}^{\alpha}+q_{I V}^{\alpha}+q_{c u r}^{\alpha} .
\end{aligned}
$$

Functions $\tilde{\rho}_{i}$ and $\tilde{T}_{i}^{\alpha \beta}$ are introduced in the following way:

$$
\begin{aligned}
& \rho \epsilon=\int d R \sum_{i=1}^{N} \delta\left(\mathbf{r}-\mathbf{r}_{i}\right) \tilde{\rho} \epsilon_{i}, \\
& T^{\alpha \beta}=\int d R \sum_{i=1}^{N} \delta\left(\mathbf{r}-\mathbf{r}_{i}\right) \tilde{T}_{i}^{\alpha \beta} .
\end{aligned}
$$

Functions $q_{0}^{\alpha}, q_{I}^{\alpha}, \ldots, q_{I V}^{\alpha}$ are associated with the order of derivative appearing before amplitude of wave function $a(R, t)$; function $q_{c u r}^{\alpha}$ is contribution in the thermal current 
Balance equations in semi-relativistic quantum hydrodynamics

from the current-current interaction. These functions are defined as

$$
\begin{aligned}
q_{0}^{\alpha}= & \int d R \sum_{i=1}^{N} \delta\left(\mathbf{r}-\mathbf{r}_{i}\right) \frac{\hbar^{2}}{4 m c^{2}} a^{2}\left[-\frac{1}{2} v_{i}^{2} \partial_{i}^{\alpha} \partial_{i}^{\beta} v_{i}^{\beta}\right. \\
& +v_{i}^{\gamma} \partial_{i}^{\alpha} v_{i}^{\beta} \partial_{i}^{\beta} v_{i}^{\gamma}-v_{i}^{\beta} \partial_{i}^{\alpha} v_{i}^{\gamma} \partial_{i}^{\beta} v_{i}^{\gamma}-v_{i}^{\beta} v_{i}^{\gamma} \partial_{i}^{\alpha} \partial_{i}^{\beta} v_{i}^{\gamma} \\
& \left.+v_{i}^{\alpha}\left[\left(\partial_{i}^{\beta} v_{i}^{\gamma}\right)\left(\partial_{i}^{\beta} v_{i}^{\gamma}\right)+v_{i}^{\beta} \Delta_{i} v_{i}^{\beta}\right]-\frac{\hbar^{2}}{4 m^{2}} \partial_{i}^{\alpha} \partial_{i}^{\beta} \Delta_{i} v_{i}^{\beta}\right], \\
q_{I}^{\alpha}= & \int d R \sum_{i=1}^{N} \delta\left(\mathbf{r}-\mathbf{r}_{i}\right) \frac{\hbar^{2}}{8 m c^{2}} a \partial_{i}^{\gamma} a\left[-2 v_{i}^{2} \partial_{i}^{\alpha} v_{i}^{\gamma}\right. \\
& \left.+4 v_{i}^{\alpha} v_{i}^{\beta} \partial_{i}^{\gamma} v_{i}^{\beta}-\frac{\hbar^{2}}{m^{2}} \partial_{i}^{\alpha} \partial_{i}^{\beta} \partial_{i}^{\gamma} v_{i}^{\beta}-\frac{\hbar^{2}}{m^{2}} \partial_{i}^{\alpha} \Delta_{i} v_{i}^{\gamma}\right] \\
q_{I I}^{\alpha}= & \int d R \sum_{i=1}^{N} \delta\left(\mathbf{r}-\mathbf{r}_{i}\right) \frac{\hbar^{2}}{4 m c^{2}}\left[-\frac{\hbar^{2}}{2 m^{2}} a \Delta_{i} a \partial_{i}^{\alpha} \partial_{i}^{\beta} v_{i}^{\beta}\right. \\
& -\frac{\hbar^{2}}{m^{2}} a \partial_{i}^{\beta} \partial_{i}^{\gamma} a \partial_{i}^{\alpha} \partial_{i}^{\beta} v_{i}^{\gamma} \\
& \left.-a^{2} \partial_{i}^{\alpha} \partial_{i}^{\beta} \ln a\left\{3 v_{i}^{\beta} v_{i}^{2}+\frac{\hbar^{2}}{2 m^{2}}\left(\partial_{i}^{\beta} \partial_{i}^{\gamma} v_{i}^{\gamma}+\Delta_{i} v_{i}^{\beta}\right)\right\}\right] \\
& \left.+2 v_{i}^{\beta} \Delta_{i} a \partial_{i}^{\alpha} \partial_{i}^{\beta} a-v_{i}^{\alpha} a \Delta_{i} \Delta_{i} a\right), \\
q_{I V}^{\alpha}= & \int d R \sum_{I I I}^{N} \delta\left(\mathbf{r}-\mathbf{r}_{i}\right) \frac{\hbar^{4}}{8 m^{3} c^{2}}\left(v_{i}^{\alpha} \Delta_{i} a \Delta_{i} a-2 v_{i}^{\beta} a^{-1} \partial_{i}^{\alpha} a \partial_{i}^{\beta} a \Delta_{i} a\right. \\
& \int d R \sum_{i=1}^{N} \delta\left(\mathbf{r}-\mathbf{r}_{i}\right) \frac{\hbar^{4}}{4 m^{3} c^{2}}\left[-a \partial_{i}^{\beta} \Delta_{i} a \partial_{i}^{\alpha} v_{i}^{\beta}\right. \\
& \left.+\left(\partial_{i}^{\alpha} a \partial_{i}^{\beta} \partial_{i}^{\gamma} a-a \partial_{i}^{\alpha} \partial_{i}^{\beta} \partial_{i}^{\gamma} a\right) \partial_{i}^{\beta} v_{i}^{\gamma}\right], \\
& \\
& \\
& \\
&
\end{aligned}
$$

and

$$
\begin{aligned}
& q_{c u r}^{\alpha}=\int d R \sum_{i, j=1, j \neq i}^{N} \delta\left(\mathbf{r}-\mathbf{r}_{i}\right) \frac{e^{2} \hbar^{2} a^{2}}{8 m^{2} c^{2}}\left[G _ { i j } ^ { \alpha \beta } \left(2 \partial_{j \beta} v_{i \gamma} a^{-1} \partial_{i \gamma} a+\partial_{j \beta} \partial_{i \gamma} v_{i \gamma}\right.\right. \\
& \left.+4 a^{-1} \Delta_{i} a v_{j \beta}\right)+G_{i j}^{\beta \gamma}\left(3 \partial_{i \alpha} v_{j \gamma} a^{-1} \partial_{i \beta} a+3 v_{j \gamma} \partial_{i \alpha} \partial_{i \beta} \ln a\right. \\
& \left.+2 \partial_{i \alpha} \partial_{i \beta} v_{j \gamma}+\partial_{i \alpha} v_{i \beta} a^{-1} \partial_{j \gamma} a-v_{i \beta} \partial_{i \alpha} \partial_{j \gamma} \ln a\right) \\
& \left.+\partial_{i \alpha} G_{i j}^{\beta \gamma}\left(\partial_{i \beta} v_{j \gamma}+2 v_{j \gamma} a^{-1} \partial_{i \beta} a\right)\right] .
\end{aligned}
$$

As a result, the energy balance equation take following form:

$$
\partial_{t} \varepsilon(\mathbf{r}, t)+\partial_{\alpha} Q^{\alpha}(\mathbf{r}, t)=e n(\mathbf{r}, t) \mathbf{v}(\mathbf{r}, t) \mathbf{E}+\mathcal{A}(\mathbf{r}, t),
$$

where functions $\varepsilon$ and $Q^{\alpha}$ are defined by formulas (711)-(73) and (75)-(84) correspondingly. Form of work density $\mathcal{A}$ is considered below in details. It is convenient to divide it on three parts: $\mathcal{A}(\mathbf{r}, t)=A^{c l}(\mathbf{r}, t)+A^{q}(\mathbf{r}, t)+\alpha(\mathbf{r}, t) . A^{c l}(\mathbf{r}, t)$ is part of work density corresponding to classical expression (see the right-hand side of equation (15)), 
$A^{q}(\mathbf{r}, t)$ is quantum part of work density:

$$
\begin{aligned}
& A^{q}(\mathbf{r}, t)=\frac{\hbar^{2} e}{4 m^{2} c^{2}}\left[\partial^{\alpha}\left(\partial^{\beta} E^{\beta} \cdot n v^{\alpha}\right)+\Delta E^{\alpha} n v^{\alpha}\right. \\
& \left.+\partial^{\alpha} E^{\beta}\left(v^{\alpha} \partial^{\beta} n+v^{\beta} \partial^{\alpha} n\right)+E^{\alpha} \partial^{\alpha} \partial^{\beta}\left(n v^{\beta}\right)\right] \\
& -\frac{\hbar^{2} e^{2}}{8 m^{2} c^{2}} \int d \mathbf{r}^{\prime} \Delta G^{\alpha \beta}\left(\mathbf{r}-\mathbf{r}^{\prime}\right)\left[n v^{\alpha}(\mathbf{r}, t) \partial_{\beta}^{\prime} n\left(\mathbf{r}^{\prime}, t\right)+n v^{\beta}\left(\mathbf{r}^{\prime}, t\right) \partial_{\alpha} n(\mathbf{r}, t)\right] .
\end{aligned}
$$

Function $\alpha(\mathbf{r}, t)$ has sense of thermal work density and can be represented as sum of three parts:

$$
\alpha(\mathbf{r}, t)=\alpha^{c l}(\mathbf{r}, t)+\alpha^{r}(\mathbf{r}, t)+\alpha^{c u r}(\mathbf{r}, t),
$$

where $\alpha^{c l}$ corresponds to classical part of thermal work density (20),

$$
\begin{aligned}
& \alpha^{r}(\mathbf{r}, t)=\int d R \sum_{i=1}^{N} \delta\left(\mathbf{r}-\mathbf{r}_{i}\right) \frac{e \hbar^{2}}{4 m^{2} c^{2}}\left[\partial_{i \alpha}\left(\partial_{i \beta} E_{i \beta} a^{2} u_{i \alpha}\right)\right. \\
& +\Delta_{i} E_{i \alpha} a^{2} u_{i \alpha}+E_{i \alpha} \partial_{i \alpha} \partial_{i \beta}\left(a^{2} u_{i \beta}\right) \\
& \left.+2 \partial_{i \alpha} E_{i \beta}\left(a \partial_{i \beta} a u_{i \alpha}+a \partial_{i \alpha} a u_{i \beta}+a^{2} \partial_{i \alpha} v_{i \beta}\right)\right]
\end{aligned}
$$

is completely quantum part of thermal work density associated with the relativistic correction to kinetic energy,

$$
\begin{aligned}
& \alpha^{c u r}(\mathbf{r}, t)=\int d R \sum_{i, j=1, j \neq i}^{N} \delta\left(\mathbf{r}-\mathbf{r}_{i}\right)\left[\frac{e^{2} \hbar^{2}}{8 m^{2} c^{2}} \partial_{i \gamma} G_{i j}^{\alpha \beta} \times\right. \\
& \quad \times\left\{v_{i \alpha} a^{2} \partial_{i \gamma} \partial_{j \beta} \ln a+v_{j \beta} a^{2} \partial_{i \gamma} \partial_{i \alpha} \ln a\right. \\
& -a\left(4 v_{i \alpha} \partial_{i \gamma} \partial_{j \beta} a+2 v_{i \gamma} \partial_{i \alpha} \partial_{j \beta} a+4 v_{j \beta} \partial_{i \gamma} \partial_{i \alpha} a\right. \\
& \left.+3 \partial_{i \gamma} v_{i \alpha} \partial_{j \beta} a+4 \partial_{i \alpha} v_{j \beta} \partial_{i \gamma} a+3 \partial_{i \gamma} v_{j \beta} \partial_{i \alpha} a+3 \partial_{i \alpha} \partial_{i \gamma} v_{j \beta} a\right) \\
& -\left[v_{i \alpha} a^{2} \partial_{j \beta} \partial_{j \gamma} \ln a+v_{j \beta} a^{2} \partial_{i \alpha} \partial_{j \gamma} \ln a\right. \\
& \left.\left.+2 v_{j \gamma} a \partial_{i \alpha} \partial_{j \beta} a+\partial_{j \gamma} v_{i \alpha} a \partial_{j \beta} a+\partial_{j \gamma} v_{j \beta} a \partial_{i \alpha} a+\partial_{j \gamma} \partial_{i \alpha} v_{j \beta} a^{2}\right]\right\} \\
& \left.-\frac{e^{2} \hbar^{2}}{4 m^{2} c^{2}} \Delta_{i} G_{i j}^{\alpha \beta}\left(a \partial_{j \beta} a u_{i \alpha}+a \partial_{i \alpha} a u_{j \beta}+a^{2} \partial_{i \alpha} v_{j \beta}\right)\right]
\end{aligned}
$$

is quantum part of thermal work density, presence of which is associated with the current-current interaction between particles. Function $\alpha^{c u r}$, as the functions of thermal energy density $n \epsilon$, quantum Bohm potential $T_{\alpha \beta}$, thermal current $q^{\alpha}$, classical part of thermal work density $\alpha^{c l}$, involve velocities of particles, which are the sum of the velocity field and the thermal velocities. It should be noticed that these quantities involve velocity field, not thermal velocities only.

Equations (55), (56) and (85) form a set of quantum hydrodynamical equations in the five-moment approximation including the continuity equation, the Euler equation and the energy balance equation.

It is necessary to notice that after introducing of the velocity field possibility to compare equations of quantum hydrodynamics with classics appears. These equations coincide to the accuracy of terms proportional to the Planck constant $\hbar$. In such a way possibility to derive quantum and classical equations at the same time. Classical 
equations obtain by turning to zero of Planck constant in the equations of quantum hydrodynamics.

In the absence of thermal contribution and many-particle quantum correlations we can present simplified form of quantum part of $\mathbf{q}(76)$, which is an analog of the quantum Bohm potential (70) in the Euler equation (29). The quantum Bohm potential is the quantum part of the flux of particle current (the momentum current in the nonrelativistic approach). Here we have the quantum part of the energy current presented as the sum of relativistic and non-relativistic parts:

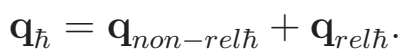

The non-relativistic quantum energy current was obtained in Ref. [1]. It can be written as

$$
q_{n o n-r e l \hbar}^{\alpha}=-\frac{\hbar^{2}}{4 m}\left(\partial_{\beta} n\right) \partial_{\alpha} v^{\beta}-\frac{\hbar^{2}}{4 m} n \partial^{\alpha}(\nabla \mathbf{v}) .
$$

The semi-relativistic part of the quantum energy current is rather large, so we present it as sum of several terms

$$
q_{r e l \hbar}^{\alpha}=q_{0, \hbar}^{\alpha}+q_{I, \hbar}^{\alpha}+q_{I I, \hbar}^{\alpha}+q_{I I I, \hbar}^{\alpha}+q_{I V, \hbar}^{\alpha},
$$

where terms are separated by order of the spatial derivatives before the particle concentration. These terms have following explicit form.

$$
\begin{aligned}
q_{0, \hbar}^{\alpha}= & \frac{\hbar^{2}}{4 m c^{2}} n\left[-\frac{1}{2} \mathbf{v}^{2} \partial^{\alpha}(\nabla \mathbf{v})\right. \\
& +v^{\gamma}\left(\partial^{\alpha} v^{\beta}\right) \partial^{\beta} v^{\gamma}-v^{\beta}\left(\partial^{\alpha} v^{\gamma}\right) \partial^{\beta} v^{\gamma}-v^{\beta} v^{\gamma} \partial^{\alpha} \partial^{\beta} v^{\gamma} \\
& \left.+v^{\alpha}\left(\partial^{\beta} v^{\gamma}\right)\left(\partial^{\beta} v^{\gamma}\right)+v^{\alpha} v^{\beta} \Delta v^{\beta}-\frac{\hbar^{2}}{4 m^{2}} \partial^{\alpha} \partial^{\beta} \Delta v^{\beta}\right]
\end{aligned}
$$

contains the particle concentration without derivatives of the particle concentration. The first six terms have the third order of the velocity field with two spatial derivatives. The last term has the first order of the velocity field, four spatial derivatives of the velocity field and additional square of the Plank constant.

$$
\begin{aligned}
q_{I, \hbar}^{\alpha}= & \frac{\hbar^{2}}{8 m c^{2}}\left(\partial^{\gamma} n\right)\left[-\mathbf{v}^{2} \partial^{\alpha} v^{\gamma}\right. \\
& \left.+2 v^{\alpha} v^{\beta} \partial^{\gamma} v^{\beta}-\frac{\hbar^{2}}{2 m^{2}} \partial^{\alpha} \partial^{\beta} \partial^{\gamma} v^{\beta}-\frac{\hbar^{2}}{2 m^{2}} \partial^{\alpha} \Delta v^{\gamma}\right]
\end{aligned}
$$

is proportional to gradient of the particle concentration. It also contains two group of terms. The first group has the third order of the velocity field with one spatial derivatives. The second group contains the first order of the velocity field with three spatial derivatives and additional square of the Plank constant.

$$
\begin{aligned}
q_{I I, \hbar}^{\alpha}= & -\frac{\hbar^{4}}{4 m^{3} c^{2}}\left[\frac{1}{2} \sqrt{n}(\Delta \sqrt{n}) \partial^{\alpha} \partial^{\beta} v^{\beta}+\sqrt{n}\left(\partial^{\beta} \partial^{\gamma} \sqrt{n}\right) \partial^{\alpha} \partial^{\beta} v^{\gamma}\right. \\
& \left.+\frac{1}{2} n\left(\partial^{\alpha} \partial^{\beta} \ln \sqrt{n}\right)\left(\partial^{\beta} \partial^{\gamma} v^{\gamma}+\Delta v^{\beta}\right)\right] \\
& -\frac{\hbar^{2}}{4 m c^{2}} 3 n\left(\partial^{\alpha} \partial^{\beta} \ln \sqrt{n}\right) v^{\beta} \mathbf{v}^{2}
\end{aligned}
$$


consists of two groups of terms. The first of them consists of three terms. All of them contain the spatial derivatives of the concentration up to the second derivative. They also contain the second derivative of the velocity field. The second group consists of the one term. It has similar dependence on order of derivatives of the particle concentration as the first group of terms. It contains the third order of the velocity field without derivatives of the velocity field. It also explicitly shows $v^{2} / c^{2}$.

$$
\begin{aligned}
q_{I I I, \hbar}^{\alpha}= & \frac{\hbar^{4}}{4 m^{3} c^{2}}\left[-\sqrt{n}\left(\partial^{\beta} \Delta \sqrt{n}\right) \partial^{\alpha} v^{\beta}\right. \\
& \left.+\left(\left(\partial^{\alpha} \sqrt{n}\right) \partial^{\beta} \partial^{\gamma} \sqrt{n}-\sqrt{n} \partial^{\alpha} \partial^{\beta} \partial^{\gamma} \sqrt{n}\right) \partial^{\beta} v^{\gamma}\right],
\end{aligned}
$$

here we find the first spatial derivatives of the velocity field along with the spatial derivatives of the particle concentration. Each term may contain product of several concentrations, some of them may be under spatial derivatives, and total order of these derivatives is three.

$$
\begin{aligned}
q_{I V, \hbar}^{\alpha}= & \frac{\hbar^{4}}{8 m^{3} c^{2}}\left(v^{\alpha}(\Delta \sqrt{n})^{2}-\frac{1}{2} v^{\beta} \frac{1}{n \sqrt{n}}\left(\partial^{\alpha} n\right)\left(\partial^{\beta} n\right)(\Delta \sqrt{n})\right. \\
& \left.+2 v^{\beta}(\Delta \sqrt{n}) \partial^{\alpha} \partial^{\beta} \sqrt{n}-v^{\alpha} \sqrt{n} \Delta \Delta \sqrt{n}\right)
\end{aligned}
$$

contains the first order of the velocity field without spatial derivatives acting on it. $\mathbf{q}_{I V, \hbar}$ has spatial derivatives of the particle concentration up to the fourth order. Formulas (90)-(97) is a step to get a closed set of the QHD equations in the five-moment approximation.

\section{Spectrum of linear excitations in semi-relativistic quantum plasmas}

Let us consider high-frequency linear excitations in semi-relativistic quantum plasmas consisting of electrons and ions (ions are assumed to be immobile). The number density and the velocity field are represented as $n=n_{0}+n^{\prime}, v_{\alpha}=0+v_{\alpha}^{\prime}, n_{0}$ is an equilibrium

value of the number density; currents of particles are supposed to be absent in the equilibrium state. At linearization hydrodynamic equations take form

$$
\begin{gathered}
\partial_{t} n^{\prime}+n_{0} \partial_{\alpha} v_{\alpha}^{\prime}=0 \\
m \partial_{t} v_{\alpha}^{\prime}-\frac{\hbar^{2}}{4 m n_{0}} \partial_{\alpha} \Delta n^{\prime}-\frac{\hbar^{4}}{8 m^{3} c^{2} n_{0}} \partial_{\alpha} \Delta \Delta n^{\prime}+\gamma \frac{T}{n_{0}} \partial_{\alpha} n^{\prime}= \\
=e\left[\partial_{\alpha} \varphi+\frac{\hbar^{2}}{4 m^{2} c^{2}} \partial_{\alpha} \Delta \varphi-\frac{5 T}{2 m c^{2}} \partial_{\alpha} \varphi-\frac{e^{2} n_{0}}{2 m c^{2}} \int d \mathbf{r}^{\prime} G_{\alpha \beta}\left(\mathbf{r}-\mathbf{r}^{\prime}\right) \partial_{\beta}^{\prime} \varphi\left(\mathbf{r}^{\prime}, t\right)\right. \\
\left.-\frac{e \hbar^{2}}{8 m^{2} c^{2}} \int d \mathbf{r}^{\prime} \partial_{\gamma} G_{\alpha \beta}\left(\mathbf{r}-\mathbf{r}^{\prime}\right) \partial_{\beta}^{\prime} \partial_{\gamma}^{\prime} n^{\prime}\left(\mathbf{r}^{\prime}, t\right)\right]
\end{gathered}
$$

and

$$
\Delta \varphi=4 \pi e n^{\prime}
$$

in the fourth term in the left-hand side of equation (99) we use equation of adiabatic process and express pressure through temperature using equation of state $p=n T$ (where 
$T$ is temperature, $\gamma$ is the adiabatic index) and assuming that wave propagation is an adiabatic process.

Carrying Fourier transformation and taking into account that $G_{\alpha \beta}(\mathbf{k})=$ $\left(8 \pi / k^{2}\right)\left(\delta_{\alpha \beta}-k_{\alpha} k_{\beta} / k^{2}\right)$, from condition of equality to zero for determinant of this set we obtain dispersion relation for the semi-relativistic Langmuir waves in plasma:

$$
\omega^{2}(k)=\omega_{p}^{2}\left(1-\frac{\hbar^{2} k^{2}}{4 m^{2} c^{2}}-\frac{5 T}{2 m c^{2}}\right)+\frac{\hbar^{2} k^{4}}{4 m^{2}}-\frac{\hbar^{4} k^{6}}{8 m^{4} c^{2}}+\frac{\gamma T}{m} k^{2},
$$

where $\omega_{p}=\sqrt{4 \pi e^{2} n_{0} / m}$ is the Langmuir frequency, $\gamma=3$ in classic adiabatic onedimensional case (see [82], chapter 4 section 4). This expression differs from classical one by presence of three terms, first of which is proportional to $k^{2} / c^{2}$ and it is consequence of new quantum semi-relativistic terms in the right-hand side of momentum balance equation. The first term proportional the Langmuir frequency contains three different contributions: the electric force giving the non-relativistic Langmuir oscillations, hybrid of the electric force and the semi-relativistic part of kinetic energy given by the last term of formula (33) or the first term in formula (57), and the thermal relativistic corrections having classic nature and presented by the first part of formula (33) correspondingly. The second and third terms are proportional to $k^{4}$ and $k^{6}$ correspondingly and arise from formula obtained above for the quantum contribution in pressure given by the quantum Bohm potential (70).

In our recent paper [40] we have considered contribution of the Darwin term in the Euler equation along with other semi-relativistic terms given by the Darwin Lagrangian and studied in this paper. We found that the hybrid force appearing at simultaneous account of the electric force and the semi-relativistic part of kinetic energy (see the first term in formula (57) ) and the force field given by the Darwin interaction partially cancel each other living no trace in linear approximation. Consequently being considered together they do not give contribution in the spectrum of Langmuir waves. Therefore final semi-relativistic spectrum of the Langmuir waves is

$$
\omega^{2}(k)=\omega_{p}^{2}\left(1-\frac{5 T}{2 m c^{2}}\right)+\frac{\hbar^{2} k^{4}}{4 m^{2}}-\frac{\hbar^{4} k^{6}}{8 m^{4} c^{2}}+\frac{\gamma T}{m} k^{2} .
$$

This result also gives generalization of the spectrum obtained in Ref. [32, where authors did not considered contribution from the relativistic part of kinetic energy in equations of collective motion. They aimed to neglect contribution from relativistic part of the quantum Bohm potential (the third term in formula (102)). However, it appears that the relativistic part of kinetic energy gives additional terms in the force field via commutators of corresponding terms in the Hamiltonian (23) and the quantum particle current (28).

\section{Conclusion}

During this work microscopic equations of quantum hydrodynamics for semi-relativistic system of particles based on the Darwin Hamiltonian. Equations were obtained in fivemoment approximation including continuity equation, balance equation for momentum 
and energy. Role of quantum relativistic terms is emphasized in the momentum and energy balance equations. Unlike the Coulomb quantum plasma, where contribution of quantum effects is reduced mainly to the quantum Bohm potential and exchange interaction, in the considered case quantum relativistic effects lead to presence of terms with complex structure in the equations. In this paper we stay on derivation of equations and, as an illustration of quantum relativistic effects we consider dispersion of proper waves in semi-relativistic quantum plasma. From obtained dispersion relation it is seen that quantum relativistic effects appear even in linear approximation. So, we may expect appearing of various nonlinear quantum relativistic phenomena, at investigation of which equations derived in this paper can be applied.

\section{Appendix: Method of the Lorentz force extraction}

Let us consider the second term in the quantum force field describing the current-current interaction $\mathcal{F}_{c u r}^{\alpha}(34)$ since it gives the magnetic part of the Lorentz force. This term appears as

$$
\begin{aligned}
& F_{L}^{\alpha}=\frac{e^{2}}{8 m^{2} c^{2}} \int d R \sum_{i, j=1, i \neq j}^{N} \delta\left(\mathbf{r}-\mathbf{r}_{i}\right)\left(\partial_{i}^{\alpha} G_{i j}^{\beta \gamma}-\partial_{i}^{\beta} G_{i j}^{\alpha \gamma}\right) \times \\
& \times\left(\psi^{*} D_{i}^{\beta} D_{j}^{\gamma} \psi+D_{i}^{* \beta} \psi^{*} D_{j}^{\gamma} \psi+c . c .\right) .
\end{aligned}
$$

We can represent it in terms of a two particle function

$$
F_{L}^{\alpha}=\frac{e^{2}}{2 m^{2} c^{2}} \int\left(\partial^{\alpha} G^{\beta \gamma}\left(\mathbf{r}-\mathbf{r}^{\prime}\right)-\partial^{\beta} G^{\alpha \gamma}\left(\mathbf{r}-\mathbf{r}^{\prime}\right)\right) \Im_{2}^{\beta \gamma}\left(\mathbf{r}, \mathbf{r}^{\prime}, t\right) d \mathbf{r}^{\prime}
$$

where

$$
\begin{aligned}
& \Im_{2}^{\beta \gamma}\left(\mathbf{r}, \mathbf{r}^{\prime}, t\right)=\int d R \sum_{i, j=1, i \neq j}^{N} \delta\left(\mathbf{r}-\mathbf{r}_{i}\right) \delta\left(\mathbf{r}^{\prime}-\mathbf{r}_{j}\right) \times \\
& \times \frac{1}{4}\left(\psi^{*} D_{i}^{\beta} D_{j}^{\gamma} \psi+D_{i}^{* \beta} \psi^{*} D_{j}^{\gamma} \psi+c . c .\right) .
\end{aligned}
$$

In the self-consistent field approximation a product of non-relativistic parts of the current $\mathbf{j}(28)$ arises in $\Im_{2}^{\alpha \beta}\left(\mathbf{r}, \mathbf{r}^{\prime}, t\right)$. So we have $\Im_{2}^{\alpha \beta}\left(\mathbf{r}, \mathbf{r}^{\prime}, t\right)=j_{N R}^{\alpha}(\mathbf{r}, t) j_{N R}^{\beta}\left(\mathbf{r}^{\prime}, t\right)$, where $\mathbf{j}_{N R}(\mathbf{r}, t)$ is the non-relativistic part of current $\mathbf{j}(28)$. Since $\Im_{2}^{\alpha \beta}\left(\mathbf{r}, \mathbf{r}^{\prime}, t\right)$ is in a semirelativistic term we can present it as $\Im_{2}^{\alpha \beta}\left(\mathbf{r}, \mathbf{r}^{\prime}, t\right)=j^{\alpha}(\mathbf{r}, t) j^{\beta}\left(\mathbf{r}^{\prime}, t\right)$, with the full currents j (28). Formula (104) now appears as

$$
\begin{aligned}
F_{L}^{\alpha} & =\frac{e^{2}}{2 m^{2} c^{2}} j^{\beta}(\mathbf{r}, t) \int\left(\partial^{\alpha} G^{\beta \gamma}\left(\mathbf{r}-\mathbf{r}^{\prime}\right)-\partial^{\beta} G^{\alpha \gamma}\left(\mathbf{r}-\mathbf{r}^{\prime}\right)\right) j^{\gamma}\left(\mathbf{r}^{\prime}, t\right) d \mathbf{r}^{\prime} \\
& =\frac{e^{2}}{2 m^{2} c^{2}} j^{\beta}(\mathbf{r}, t)\left[\partial^{\alpha} \int G^{\beta \gamma}\left(\mathbf{r}-\mathbf{r}^{\prime}\right) j^{\gamma}\left(\mathbf{r}^{\prime}, t\right) d \mathbf{r}^{\prime}\right. \\
& \left.-\partial^{\beta} \int G^{\alpha \gamma}\left(\mathbf{r}-\mathbf{r}^{\prime}\right) j^{\gamma}\left(\mathbf{r}^{\prime}, t\right) d \mathbf{r}^{\prime}\right] .
\end{aligned}
$$

Introducing the vector potential of magnetic field created by moving charges

$$
A_{\text {int }}^{\alpha}(\mathbf{r}, t)=\frac{e}{2 m c} \int G^{\alpha \beta}\left(\mathbf{r}-\mathbf{r}^{\prime}\right) j^{\beta}\left(\mathbf{r}^{\prime}, t\right) d \mathbf{r}^{\prime},
$$


and using the following identity

$$
\partial^{\alpha} A_{i n t}^{\beta}-\partial^{\beta} A_{i n t}^{\alpha}=\varepsilon^{\alpha \beta \gamma} B_{i n t}^{\gamma}
$$

(it is meaning $\mathbf{B}_{\text {int }}=$ curl $\mathbf{A}_{\text {int }}$ ), we find

$$
F_{L}^{\alpha}=\frac{e}{m c} \varepsilon^{\alpha \beta \gamma} j^{\beta} B_{i n t}^{\gamma} .
$$

Applying $\mathbf{j}=m n \mathbf{v}$ (see formula (54)) we can represent $\mathbf{F}_{L}$ in the final form

$$
\mathbf{F}_{L}=\frac{e}{c} n\left[\mathbf{v}, \mathbf{B}_{\text {int }}\right] \text {. }
$$

Let us admit that magnetic field $\mathbf{B}_{\text {int }}$ obeys the magneto-static Maxwell equations $\operatorname{curl} \mathbf{B}_{\text {int }}=\frac{4 \pi}{c} \mathbf{j}\left(\underline{65)}\right.$ and $\operatorname{div} \mathbf{B}_{\text {int }}=0$ (64).

\section{References}

[1] L. S. Kuzmenkov, S. G. Maksimov, Theor. Math. Phys. 118, N20, 227 (1999).

[2] L. S. Kuzmenkov, S. G. Maksimov, V. V. Fedoseev, Theor. Math. Phys. 126, N1, 110 (2001).

[3] P. A. Andreev, L. S. Kuzmenkov, M. I. Trukhanova, Phys. Rev. B 84, 245401 (2011).

[4] P. A. Andreev, L. S. Kuz'menkov, Int. J. Mod. Phys. B 26, N32, 1250186 (2012).

[5] P. A. Andreev, L. S. Kuz'menkov, Rus. J. Phys. 50, N12, 1251 (2007).

[6] L. D. Landau, E. M. Lifshitz, The Classical Theory of Fields (Butterworth-Heinemann, 1975).

[7] A. A. Vlasov, Sov. Phys. Usp. 10, 721 (1968).

[8] D. Bohm, E. P. Gross, Phys. Rev. 75, 1851 (1949).

[9] L. P. Pitaevskii, E. M. Lifshitz, Physical Kinetics (Pergamon Press, 1981).

[10] Yu. L. Klimontovich, Statistical Physics [in Russian], Nauka, Moscow (1982); English transl., Harwood, New York (1986).

[11] G. M. Zaslavskii, PMTF N5, 42 (1962). (in Russian)

[12] I. P. Pavlotskii, DAN USSR 213, N4, 812 (1973). (in Russian)

[13] Yu. N. Orlov, I. P. Pavlotsky, Matem. Mod. 1:12, 31 (1989).

[14] Yu. N. Orlov, I. P. Pavlotsky. Physica A 158, 607 (1989).

[15] Yu. N. Orlov, V. V. Vedenyapin, Mod. Phys. Lett. B, 9, N5, 291 (1995).

[16] Yu. N. Orlov, I. P. Pavlotsky, Physica A 184, 558 (1992).

[17] O. V. Mingalev, Yu. N. Orlov, V. V. Vedenyapin, Phys. Lett. A 223, 246 (1996).

[18] P. Goldstein, L. A. Turski, Physica 89A, 481 (1977).

[19] R. D. Jones, Phys. Fluids 21, 2186 (1978).

[20] R. D. Jones, Phys. Fluids 21, 2191 (1978).

[21] R. D. Jones, A. Pytte, Phys. Fluids 23, 269 (1980).

[22] R. D. Jones, A. Pytte, Phys. Fluids 23, 273 (1980).

[23] M. Hillery, R.F. O'Connell, M.O. Scully, E.P. Wigner, Physics Reports, 106, 121 (1984).

[24] H. A. Shah, W. Masood, M. N. S. Qureshi, and N. L. Tsintsadze, Phys. Plasmas 18, 102306 (2011).

[25] M. Akbari-Moghanjoughi, Phys. Plasmas 20, 042706 (2013).

[26] S. M. Mahajan, Phys. Rev. Lett. 90, 035001 (2003).

[27] F. A. Asenjo, F. A. Borotto, Abraham C.-L. Chian, V. Munoz, J. A. Valdivia, E. L. Rempel, Phys. Rev. E 85, 046406 (2012).

[28] R. A. Lopez, F. A. Asenjo, V. Munoz, A. C.-L. Chian, and J. A. Valdivia, Phys. Rev. E 88, 023105 (2013).

[29] C. Ruyer, L. Gremillet, D. Benisti, and G. Bonnaud, Phys. Plasmas 20, 112104 (2013).

[30] P. A. Andreev, arXiv:1208.0998.

[31] S. M. Mahajan, Z. Yoshida, Phys. Plasm. 18, 055701 (2011).

[32] F. A. Asenjo, J. Zamanian, M. Marklund, G. Brodin, P. Johansson, New J. Phys. 14, 073042 (2012). 
[33] P. A. Andreev, L. S. Kuzmenkov, Dinamika Slozhnykh Sistem (2009) (in Russian).

[34] P. A. Andreev and L. S. Kuzmenkov, PIERS Proceedings, Marrakesh, Morocco, March 20-23, p. 1047 (2011).

[35] M. I. Trukhanova, Eur. Phys. J. D 67, Issue 2, 32 (2013).

[36] M. I. Trukhanova, Int. J. Mod. Phys. B 26, N1, 1250004 (2012).

[37] F. Haas, B. Eliasson, P. K. Shukla, Phys. Rev. E 85, 056411 (2012).

[38] F. A. Asenjo, V. Munoz, J. A. Valdivia, S. M. Mahajan, Phys. Plasm. 18, 012107 (2011).

[39] D. A. Uzdensky and S. Rightley, arXiv:1401.5110.

[40] A. Yu. Ivanov, P. A. Andreev, L. S. Kuz'menkov, arXiv: 1209.6124.

[41] A. Yu. Ivanov and P. A. Andreev, Russ. Phys. J. 56, 325 (2013).

[42] G. Breit, Phys. Rev. 34, 553 (1929).

[43] V. B. Berestetskii, E. M. Lifshitz, L. P. Pitaevskii, Quantum Electrodynamics (ButterworthHeinemann, 1982).

[44] P. A. Andreev, L. S. Kuzmenkov, Phys. Rev. A 78, 053624 (2008).

[45] F. Haas. Phys. Plasm. 12, 062117 (2005).

[46] M. Marklund, G. Brodin, Phys. Rev. Lett. 98, 025001 (2007).

[47] G. Brodin, M. Marklund, New J. Phys. 9, 277 (2007).

[48] E. Madelung, Z. Phys. 40, 332 (1926).

[49] T. Takabayashi, Progr. Theor. Phys. 14, 283 (1955).

[50] F. Haas, G. Manfredi, M. Feix, Phys. Rev. E 62, 2763(2000).

[51] L. S. Kuz'menkov, S. G. Maksimov, and V. V. Fedoseev, Theor. Math. Fiz. 126258 (2001) [Theoretical and Mathematical Physics, 126212 (2001)].

[52] P. K. Shukla, B. Eliasson, Phys. Usp. 53, 51 (2010).

[53] P. K. Shukla, B. Eliasson, Rev. Mod. Phys. 83, 885 (2011).

[54] M. V. Kuzelev, A. A. Rukhadze, Phys. Usp. 42, 603 (1999).

[55] P. A. Andreev, L. S. Kuz'menkov, Physics of Atomic Nuclei 71, N.10, 1724 (2008).

[56] P. A. Andreev, L. S. Kuz'menkov, Eur. Phys. J. D 67, 216 (2013).

[57] P. A. Andreev, L.S. Kuz'menkov, Moscow University Physics Bulletin 62, N.5, 271 (2007).

[58] G. Brodin, M. Marklund, J. Zamanian, B. Ericsson and P. L. Mana, Phys. Rev. Lett. 101, 245002 (2008).

[59] D. V. Vagin, P. A. Polyakov, and N. E. Rusakova, Moscow University Physics Bulletin, 64, 133 (2009).

[60] S. V. Vladimirov, Yu. O. Tyshetsky, Physics-Uspekhi 54, 12 (2011).

[61] G. Manfredi, Fields Inst. Commun. 46, 263 (2005).

[62] G. Manfredi and F. Haas, Phys. Rev. B 64, 075316 (2001).

[63] M. Marklund, J. Zamanian, G. Brodin, Transport Theory and Statistical Physics, 39, 502 (2011).

[64] G. Brodin, M. Marklund, J. Zamanian and M. Stefan, Plasma Phys. Control. Fusion 53, 074013 (2011).

[65] M. Stefan, J. Zamanian, G. Brodin, A. P. Misra, and M. Marklund, Phys. Rev. E 83, 036410 (2011).

[66] F. Haas, M. Marklund, G. Brodin, J. Zamanian, Phys. Lett. A 374, 481 (2010).

[67] J. Zamanian, M. Stefan, M. Marklund, and G. Brodin, Phys.Plasmas 17, 102109 (2010).

[68] J. Zamanian, M. Marklund and G. Brodin, New J. Phys. 12, 043019 (2010).

[69] F. Haas, J. Zamanian, M. Marklund and G. Brodin, New J. Phys. 12, 073027 (2010).

[70] M. Trovato, and L. Reggiani, J. Phys. A: Math. Theor. 43, 102001 (2010).

[71] M. Trovato, and L. Reggiani, Phys. Rev. E 81, 021119 (2010).

[72] M. V. Altaisky, Phys. Lett. A, 374, 522 (2010).

[73] L. S. Kuz'menkov and S. G. Maksimov, Theoretical and Mathematical Physics 131641 (2002).

[74] L. S. Kuz'menkov and S. G. Maksimov, Theoretical and Mathematical Physics 143, 821 (2005).

[75] P. A. Andreev, arXiv:1308.3715.

[76] A. Yu. Ivanov, L. S. Kuz'menkov, arXiv:1308.1966. 
[77] T. Koide, Phys. Rev. C 87, 034902 (2013).

[78] M. A. Drofa, L. S. Kuzmenkov, Theor. Math. Phys. 108, N1, 849 (1996).

[79] L. S. Kuz'menkov, Theoretical and Mathematical Physics 86, 159 (1991).

[80] P. A. Andreev and L. S. Kuzmenkov, PIERS Proceedings, Moscow, Russia, August 19-23, p. 158 (2012).

[81] L. D. Landau, E. M. Lifshitz, Quantum Mechanics: Non-Relativistic Theory. Vol. 3 (3rd ed.). Pergamon Press, (1977).

[82] N. A. Krall, A. V. Trivelpiece, Principles of Plasma Physics (McGraw-Hill Book Company, 1973). 